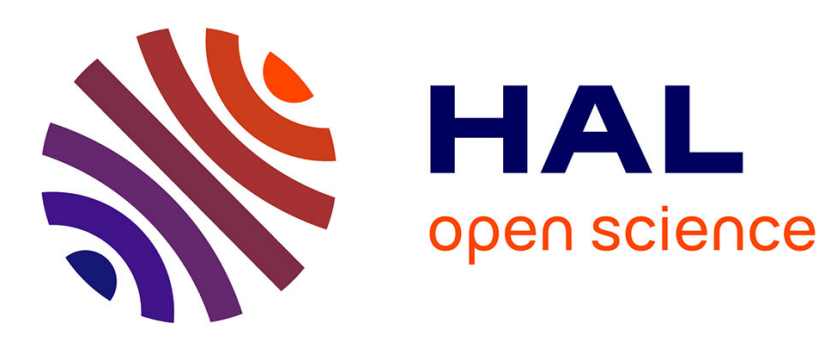

\title{
Algebra of transversely isotropic sixth order tensors and solution to higher order inhomogeneity problems
}

Vincent Monchiet, Guy Bonnet

\section{To cite this version:}

Vincent Monchiet, Guy Bonnet. Algebra of transversely isotropic sixth order tensors and solution to higher order inhomogeneity problems. Journal of Elasticity, 2013, 110 (2), pp.159-183. hal-00734420

\author{
HAL Id: hal-00734420 \\ https://hal.science/hal-00734420
}

Submitted on 21 Sep 2012

HAL is a multi-disciplinary open access archive for the deposit and dissemination of scientific research documents, whether they are published or not. The documents may come from teaching and research institutions in France or abroad, or from public or private research centers.
L'archive ouverte pluridisciplinaire HAL, est destinée au dépôt et à la diffusion de documents scientifiques de niveau recherche, publiés ou non, émanant des établissements d'enseignement et de recherche français ou étrangers, des laboratoires publics ou privés. 


\title{
Algebra of transversely isotropic sixth order tensors and solution to higher order inhomogeneity problems
}

\author{
V. Monchiet · G. Bonnet
}

Received: date / Accepted: date

\begin{abstract}
In this paper we provide a complete and irreducible representation for transversely isotropic sixth order tensors having minor symmetries. Such tensors appear in some practical problems of elasticity for which their inversion is required. For this kind of tensors, we provide an irreducible basis which possesses some remarkable properties, allowing us to provide a representation in a compact form which uses two scalars and three matrices of dimension 2,3 and 4 . It is shown that the calculation of sum, product and inverse of transversely isotropic sixth order tensors is greatly simplified by using this new formalism and appears to be appropriate for deriving new various solutions to some practical problems in mechanics which use such kinds of higher order tensors. For instance, we derive the fields within a cylindrical inhomogeneity submitted to remote gradient of strain. The method of resolution uses the Eshelby equivalent inclusion method extended to the case of a polynomial type eigenstrain. It is shown that the approach leads to a linear system involving a sixth order tensor whose closed form solution is derived by means of the tensorial formalism introduced in the first part of the paper.
\end{abstract}

Keywords Sixth order tensor · Anisotropy · Inhomogeneity problem · Cylindrical inclusions

Université Paris-Est, Laboratoire Modélisation et Simulation Multi Echelle, MSME UMR8208 CNRS, 5 boulevard Descartes, 77454 Marne la Vallée Cedex, France

Tel.: +33 (0) 160957793

Fax: +33 (0) 160957799

E-mail: vincent.monchiet@univ-paris-est.fr 


\section{Introduction}

The characterization and classification of the material symmetries ${ }^{1}$ for an anisotropic fourth order tensor have been investigated by several researchers, notably by Love [12], Voigt [30], Gurtin [10] and Thurston [28], Nye [21], Hou and Del Pierro [11], Forte and Vianello [8], Chadwick et al. [4] among many others. In these studies, the authors express the different forms of the elasticity tensor into a matrix of dimension $6 \times 6$ (by means of Voigt type notations) in the cartesian system. In the paper of Walpole [32] and later in [3], coordinate free representations of the elasticity tensors are derived by means of irreducible bases which prove to have interesting properties for the tensorial calculus. Indeed, by inspecting the properties of the multiplication table associated to these irreducible bases, symbolic representations which use scalars and matrices have been provided and shown to be appropriate for performing the standard tensorial operations such as the multiplication between two tensors and the inversion of a fourth order tensor. These "symbolic" or "compact" representations are very useful and suitable for deriving the solutions of elasticity problems for which the multiplication or more specially the inversion of fourth order tensors are required.

Some fundamental theories as well as practical problems in the area of mechanics require the introduction of tensors whose order is higher than 4. For instance, the theories of gradient elasticity or the theory of microstructures in elasticity (see Toupin [29], Mindlin [13], Mindlin \& Eshel [14], Suiker and Chang [26]) introduce gradient elastic tensors of order $5,6, \ldots$ in addition to the commonly used fourth order elasticity tensor. The use of such high order tensors raises a number of fundamental questions, especially concerning their inversion or the condition of positiveness when computing the elastic energy from these tensors. On the other hand, solutions to special elasticity problems, such as the solution to higher order inhomogeneity problems, require the inversion of tensors of order 6, 8, etc. (see Sendeckyj [22], Moschovidis [17], Moschovidis and Mura [18], Asaro and Barnett [1], Furuhashi and Mura, [9], Mura [19]). Although the tensorial equation produced in this case can be solved numerically, it is obviously of interest to derive closed form solutions which can cover many applications. Inverting such tensors involves the algebra of these higher order tensors. On the other hand, an irreducible basis for isotropic sixth and eighth order tensors having the minor symmetries ${ }^{2}$ and a closed-form solution of higher order inhomogeneity problems have been recently provided by Monchiet and Bonnet [15]. However, the hypothesis of isotropy restricts the applicability of this new formalism for handling the problem of a spherical inhomogeneity. In this paper we propose to extend these results to the case of transversely isotropic sixth order tensors. We derive an irreducible basis,

1 There are various and non equivalent definitions of the symmetry classes. Forte and Vianello [8], Chadwick et al. [4] introduced only eight symmetry classes while Hou and Del Pierro [11] introduced ten symmetry classes.

2 The definition of minor symmetries for sixth order tensors is given in section 2 and is defined by Monchiet and Bonnet [15] for tensors of order higher than 6 . 
constituted of 31 elements, for any transversely isotropic sixth order tensors. The proposed representation is coordinate free since all these tensors are constructed as the outer products of elementary tensors attached to the direction of transverse isotropy. The multiplication tables are provided in the paper as well as a compact representation which uses two scalars and three matrices of dimension 2, 3 and 4. The efficiency of this new formalism and its ability to derive closed form solution to linear elasticity problems is thereafter illustrated in the case of a cylindrical inhomogeneity subjected to a remote uniform gradient of strain.

The paper is organized as follows:

- Section 2 states about the definition of transversely isotropic tensors and addresses the problem related to their inversion.

- In section 3, we prove that the dimension of a transversely isotropic sixth order tensor is 31 .

- Section 4 then proposes an irreducible basis for such tensors and provides the multiplication tables as well as the compact representation.

- Section 5 expounds the problem of an ellipsoidal inhomogeneity subjected to a gradient of strain and its method of resolution based on the Eshelby equivalent inclusion problem. Closed form solutions are then provided for the cylindrical inhomogeneity.

Below are provided some specific notations used in the paper:

a tensor of rank 1 ,

a tensor of rank 2 and 3,

A tensor of rank 4 and 6 ,

$\otimes \quad$ tensorial product between two tensors,

$\odot_{n} \quad n^{t h}$ contraction between two tensors $\left(\right.$ ex: $\left.\left(\mathbb{A} \odot_{3} \boldsymbol{a}\right)_{i j k}=A_{i j k p q r} a_{p q r}\right)$.

\section{Statement of the problem}

Consider the following linear relation:

$$
\mathbb{A} \odot_{n} \boldsymbol{a}=\boldsymbol{b}
$$

involving two tensors $\boldsymbol{a}$ and $\boldsymbol{b}$ of order $n$ and a $2 n^{t h}$ order tensor $\mathbb{A}$. In (1), the symbol " $\odot_{n}$ " represents the $n^{\text {th }}$ contraction between two tensors such that $\left(\mathbb{A} \odot_{n} \boldsymbol{a}\right)_{i . . j}=A_{i . . j p . . q} a_{p . . q}$. In this paper we only examine the cases $n=2$ and $n=3$.

In the case $n=2$, tensors $\boldsymbol{a}$ and $\boldsymbol{b}$ are symmetric second order tensors, $a_{i j}=a_{j i}$ and $b_{i j}=b_{j i}$. Tensor $\mathbb{A}$ is a fourth order tensor having the minor symmetries, $A_{i j p q}=A_{j i p q}=A_{i j q p}$, but not the major symmetry $A_{i j p q} \neq A_{p q i j}$.

For the case $n=3$, tensors $\boldsymbol{a}$ and $\boldsymbol{b}$ are third order tensors which are symmetric according to their first two indices: $a_{i j k}=a_{j i k}$ and $b_{i j k}=b_{j i k}$. Due to the symmetries of $\boldsymbol{a}$ and $\boldsymbol{b}, A_{i j k p q r}$ is invariant by any permutation of indices 
$(i, j)$, and $(p, q)$; these symmetries are called minor symmetries for the sixth order tensor. A tensor of components $A_{i \ldots j}$ in the cartesian frame $\left(\underline{e}_{1}, \underline{e}_{2}, \underline{e}_{3}\right)$ is assumed symmetric with respect to the linear transformation $R$ if:

$$
A_{i \ldots j}=R_{i p} \ldots R_{j q} A_{p \ldots q} .
$$

We assume that:

- $\mathbb{A}$ is invariant by the symmetry with respect to the plane $O x_{1} x_{2}$ characterized by the transformation of components $R_{i j}=\delta_{i j}-2 n_{i} n_{j}$ and $\underline{n}$ is the vector $\underline{e}_{3}$. - $A_{i . . j}$ is invariant through any rotation by an angle of $\theta$ around the axis $O x_{3}$. In other words, $A_{i j k p q r}$ remains unchanged under the transformation (2) where the components of the matrix $R_{i j}$ are given by:

$$
R_{i j}=\left(\begin{array}{ccc}
\cos (\theta) & \sin (\theta) & 0 \\
-\sin (\theta) & \cos (\theta) & 0 \\
0 & 0 & 1
\end{array}\right) .
$$

With the above properties of invariance, tensor $\mathbb{A}$ is transversely isotropic and we denote by $E_{2 n}$ the space of such tensors (with $n=2$ for fourth order tensors and $n=3$ for sixth order tensors).

For the case $n=2$, tensor $\mathbb{A}$ is defined by 6 independent coefficients. Providing a basis in the case of such symmetric tensors has been effected in [3]. However, the product of two such symmetric tensors is not generally symmetric and it is therefore important to study simultaneously symmetric and unsymmetric tensors. So, an irreducible basis for such tensors has been first provided in [31] and later in [32]. In the following, we recall the principal results about this decomposition.

Any fourth order tensor $\mathbb{A} \in E_{4}$ can be constructed by the linear combination:

$$
\mathbb{A}=\sum_{n=1}^{n=6} a_{n} \mathbb{T}_{n}
$$

where the components of tensors $\mathbb{T}_{n}$ for $n=1 . .6$ are given by:

$$
\left\{\begin{array}{l}
{\left[\mathbb{T}_{1}\right]_{i j p q}=\left(\pi_{i p} \pi_{j q}+\pi_{i q} \pi_{j p}-\pi_{i j} \pi_{p q}\right) / 2} \\
{\left[\mathbb{T}_{2}\right]_{i j p q}=\left(\pi_{i p} n_{j} n_{q}+\pi_{i q} n_{j} n_{p}+\pi_{j p} n_{i} n_{q}+\pi_{j q} n_{i} n_{p}\right) / 2} \\
{\left[\mathbb{T}_{3}\right]_{i j p q}=\pi_{i j} \pi_{p q} / 2} \\
{\left[\mathbb{T}_{4}\right]_{i j p q}=\pi_{i j} n_{p} n_{q} / \sqrt{2}} \\
{\left[\mathbb{T}_{5}\right]_{i j p q}=n_{i} n_{j} \pi_{p q} / \sqrt{2}} \\
{\left[\mathbb{T}_{6}\right]_{i j p q}=n_{i} n_{j} n_{p} n_{q}}
\end{array}\right.
$$


where $\pi_{i j}$ is defined by:

$$
\pi_{i j}=\delta_{i j}-n_{i} n_{j}
$$

and $n_{i}$ are the components of the normal unit vector $\underline{n}$ defining the direction orthogonal to the plane of transverse isotropy. The double contraction between two tensors taken from $\left(\mathbb{T}_{1}, . ., \mathbb{T}_{6}\right)$ is provided in table 1 .

\begin{tabular}{|c|c|c|c|c|c|c|}
\hline$\odot_{2}$ & $\mathbb{T}_{1}$ & $\mathbb{T}_{2}$ & $\mathbb{T}_{3}$ & $\mathbb{T}_{4}$ & $\mathbb{T}_{5}$ & $\mathbb{T}_{6}$ \\
\hline $\mathbb{T}_{1}$ & $\mathbb{T}_{1}$ & 0 & 0 & 0 & 0 & 0 \\
\hline $\mathbb{T}_{2}$ & 0 & $\mathbb{T}_{2}$ & 0 & 0 & 0 & 0 \\
\hline $\mathbb{T}_{3}$ & 0 & 0 & $\mathbb{T}_{3}$ & $\mathbb{T}_{4}$ & 0 & 0 \\
\hline $\mathbb{T}_{4}$ & 0 & 0 & 0 & 0 & $\mathbb{T}_{3}$ & $\mathbb{T}_{4}$ \\
\hline $\mathbb{T}_{5}$ & 0 & 0 & $\mathbb{T}_{5}$ & $\mathbb{T}_{6}$ & 0 & 0 \\
\hline $\mathbb{T}_{6}$ & 0 & 0 & 0 & 0 & $\mathbb{T}_{5}$ & $\mathbb{T}_{6}$ \\
\hline
\end{tabular}

Table 1: The double contraction between the $\mathbb{T}_{n}$ for $n=1 . .6$

It can be observed that the set of tensors $\left\{\mathbb{T}_{1}, \mathbb{T}_{2}, \mathbb{T}_{3}, \mathbb{T}_{4}, \mathbb{T}_{5}, \mathbb{T}_{6}\right\}$ is constituted of three groups which are independents for the composition $\odot_{2}$, they are: $\left\{\mathbb{T}_{1}\right\},\left\{\mathbb{T}_{2}\right\},\left\{\mathbb{T}_{3}, \mathbb{T}_{4}, \mathbb{T}_{5}, \mathbb{T}_{6}\right\}$. Note that tensor $\mathbb{A} \in E_{4}$ has the major symmetry only if $a_{4}=a_{5}$ in (4). The table shows clearly that the product of two symmetric tensors is generally not symmetric. For completeness, we provide the relations giving the coefficients $a_{1}, . ., a_{6}$ in (4) as function of the components of tensor $\mathbb{A}$ :

$$
\begin{aligned}
& a_{1}=A_{i j p q} \pi_{i p} \pi_{j q} / 2-A_{i j p q} \pi_{i j} \pi_{p q} / 4, \quad a_{2}=A_{i j p q} \pi_{i p} n_{j} n_{q}, \\
& a_{3}=A_{i p j q} \pi_{i p} \pi_{j q} / 2, \quad a_{4}=A_{i j p q} \pi_{p q} n_{i} n_{j} / \sqrt{2}, \\
& a_{5}=A_{i j p q} \pi_{i j} n_{p} n_{q} / \sqrt{2}, \quad a_{6}=A_{i j p q} n_{i} n_{j} n_{p} n_{q} .
\end{aligned}
$$

Denoting $\boldsymbol{T}^{n}(\boldsymbol{a})=\mathbb{T}_{n} \odot_{2} \boldsymbol{a}$ for $n=1 . .6$, their components are:

$$
\left\{\begin{array}{l}
T_{i j}^{1}(\boldsymbol{a})=\pi_{i p} \pi_{j q} a_{p q}-\pi_{i j} a_{p q} \pi_{p q} / 2, \\
T_{i j}^{2}(\boldsymbol{a})=\left(\pi_{i p} n_{j}+\pi_{j p} n_{i}\right) a_{p q} n_{q}, \\
T_{i j}^{3}(\boldsymbol{a})=\pi_{i j} a_{p q} \pi_{p q} / 2, \\
T_{i j}^{4}(\boldsymbol{a})=\pi_{i j} a_{p q} n_{p} n_{q} / \sqrt{2}, \\
T_{i j}^{5}(\boldsymbol{a})=n_{i} n_{j} a_{p q} \pi_{p q} / \sqrt{2}, \\
T_{i j}^{6}(\boldsymbol{a})=n_{i} n_{j} a_{p q} n_{p} n_{q} .
\end{array}\right.
$$


It follows that the linear relation (1) for $n=2$, can be rewritten into the form:

$$
\left\{\begin{array}{l}
\boldsymbol{T}^{1}(\boldsymbol{a})=a_{1} \boldsymbol{T}^{1}(\boldsymbol{a}), \\
\boldsymbol{T}^{2}(\boldsymbol{a})=a_{2} \boldsymbol{T}^{2}(\boldsymbol{a}), \\
\boldsymbol{T}^{3}(\boldsymbol{a})=a_{3} \boldsymbol{T}^{3}(\boldsymbol{a})+a_{4} \boldsymbol{T}^{4}(\boldsymbol{a}), \\
\boldsymbol{T}^{6}(\boldsymbol{a})=a_{5} \boldsymbol{T}^{5}(\boldsymbol{a})+a_{6} \boldsymbol{T}^{6}(\boldsymbol{a})
\end{array}\right.
$$

where the $a_{n}$ for $n=1 . .6$ are the components of tensor $\mathbb{A}$ along the basis $\left(\mathbb{T}_{1}, \ldots, \mathbb{T}_{6}\right)$. Owing to these results, it appears that the components of tensor $\mathbb{A}$ can be represented by two scalars and a $2 \times 2$ matrix. This compact representation is written into the symbolic form: $\mathbb{A}=\left\{a_{1}, a_{2}, A\right\}$ where the matrix $A$ is defined by:

$$
A=\left[\begin{array}{ll}
a_{3} & a_{4} \\
a_{5} & a_{6}
\end{array}\right] .
$$

It turns out that this symbolic notation $\mathbb{A}=\left\{a_{1}, a_{2}, A\right\}$ is useful for performing the standard tensorial operations. For instance, let us introduce a second tensor $\mathbb{B}$, we denote by $b_{1}, . . b_{6}$ its components along the basis $\left(\mathbb{T}_{1}, \ldots, \mathbb{T}_{6}\right)$ and $\left\{b_{1}, b_{2}, B\right\}$ its compact representation. The product between $\mathbb{A}$ and $\mathbb{B}$ defines the fourth order tensor $\mathbb{C}=\mathbb{A} \odot{ }_{2} \mathbb{B}$ whose components are $\mathbb{C}=\left\{c_{1}=a_{1} b_{1}, c_{2}=\right.$ $\left.a_{2} b_{2}, C=A . B\right\}$ where $A . B$ represents the standard matrix product between $A$ and $B$. The inverse of tensor $\mathbb{A}$ is simply obtained by inverting the elements of its compact representation: $\mathbb{A}^{-1}=\left\{1 / a_{1}, 1 / a_{2}, A^{-1}\right\}$ where $A^{-1}$ denotes the inverse of the $2 \times 2$ matrix.

Such a representation is useful for doing the classical tensorial operations and is convenient for many practical problems in mechanics such as Eshelby's inhomogeneity problem [5]. In the following section, we aim at deriving a similar representation for transversely isotropic sixth order tensors.

\section{Dimension of the space containing transversely isotropic and symmetric sixth order tensors}

A key point in determining the representation of a tensor of a specific type by an irreducible basis lies in the calculation of the total number of elements of the basis. In this section, we address a general method for computing the dimension of the space containing sixth order tensors having the minor symmetries and we provide results for the particular case of the transversely isotropic symmetry.

As effected for the case of fourth order tensors, Voigt type notations are suitable for representing such tensors by a $6 \times 6$ matrice. In the following, we extend it to the case of a sixth order tensor. This is achieved by a vector representation 
of third-order tensors. Any third order tensor $\boldsymbol{a}$, which is symmetric according to its two first indices $\left(a_{i j k}=a_{j i k}\right)$ depends on 18 independent components and can the been represented along tensors $\boldsymbol{v}_{n}$ for $n=1 . .18$ which are defined by :

$$
\left\{\begin{array}{lll}
\boldsymbol{v}_{1}=\boldsymbol{f}_{1} \otimes \underline{e}_{1}, & \boldsymbol{v}_{2}=\boldsymbol{f}_{2} \otimes \underline{e}_{1}, & \boldsymbol{v}_{3}=\boldsymbol{f}_{3} \otimes \underline{e}_{1}, \\
\boldsymbol{v}_{4}=\boldsymbol{f}_{6} \otimes \underline{e}_{2}, & \boldsymbol{v}_{5}=\boldsymbol{f}_{5} \otimes \underline{e}_{3}, & \boldsymbol{v}_{6}=\boldsymbol{f}_{2} \otimes \underline{e}_{2}, \\
\boldsymbol{v}_{7}=\boldsymbol{f}_{1} \otimes \underline{e}_{2}, & \boldsymbol{v}_{8}=\boldsymbol{f}_{3} \otimes \underline{e}_{2}, & \boldsymbol{v}_{9}=\boldsymbol{f}_{6} \otimes \underline{e}_{1}, \\
\boldsymbol{v}_{10}=\boldsymbol{f}_{4} \otimes \underline{e}_{3}, & \boldsymbol{v}_{11}=\boldsymbol{f}_{3} \otimes \underline{e}_{3}, & \boldsymbol{v}_{12}=\boldsymbol{f}_{1} \otimes \underline{e}_{3}, \\
\boldsymbol{v}_{13}=\boldsymbol{f}_{2} \otimes \underline{e}_{3}, & \boldsymbol{v}_{14}=\boldsymbol{f}_{5} \otimes \underline{e}_{1}, & \boldsymbol{v}_{15}=\boldsymbol{f}_{4} \otimes \underline{e}_{2}, \\
\boldsymbol{v}_{16}=\boldsymbol{f}_{4} \otimes \underline{e}_{1}, & \boldsymbol{v}_{17}=\boldsymbol{f}_{5} \otimes \underline{e}_{2}, & \boldsymbol{v}_{18}=\boldsymbol{f}_{6} \otimes \underline{e}_{3}
\end{array}\right.
$$

where $\underline{e}_{1}, \underline{e}_{2}, \underline{e}_{3}$ are the unit vectors of the cartesian basis while the second order tensors $\boldsymbol{f}_{n}$ for $n=1 . .6$ are defined by :

$$
\left\{\begin{array}{lll}
\boldsymbol{f}_{1}=\underline{e}_{1} \otimes \underline{e}_{1}, & \boldsymbol{f}_{2}=\underline{e}_{2} \otimes \underline{e}_{2}, & \boldsymbol{f}_{3}=\underline{e}_{3} \otimes \underline{e}_{3}, \\
\boldsymbol{f}_{4}=\sqrt{2} \underline{e}_{2} \otimes_{s} \underline{e}_{3}, & \boldsymbol{v}_{5}=\sqrt{2} \underline{e}_{1} \otimes_{s} \underline{e}_{3}, & \boldsymbol{f}_{6}=\sqrt{2} \underline{e}_{1} \otimes_{s} \underline{e}_{2}
\end{array}\right.
$$

where $\otimes_{s}$ represents the symmetrized tensorial product of two vectors: $\underline{u} \otimes_{s} \underline{v}=$ $(\underline{u} \otimes \underline{v}+\underline{v} \otimes \underline{u}) / 2$. With the above definitions for tensors $\boldsymbol{v}_{n}$, the basis $\boldsymbol{v}_{1} . . \boldsymbol{v}_{18}$ is orthonormal for the composition " $\odot_{3}$ " since :

$$
\boldsymbol{v}_{r} \odot_{3} \boldsymbol{v}_{s}=\delta_{r s}
$$

for any $r, s=1 . .18$. In relation (1) (for the case $n=3$ ), tensors $\boldsymbol{a}, \boldsymbol{b}$ and $\mathbb{A}$ are decomposed along the basis $\boldsymbol{v}_{n}$ for $n=1 . .18$ :

$$
\boldsymbol{a}=a_{I} \boldsymbol{v}_{I}, \quad \boldsymbol{b}=b_{I} \boldsymbol{v}_{I}, \quad \mathbb{A}=A_{I J} \boldsymbol{v}_{I} \otimes \boldsymbol{v}_{J}
$$

By convention the upper case indices vary from 1 to 18 . Einstein summation convention for repeated indices is applied in the above expressions. For instance $a_{I} \boldsymbol{v}_{I}=a_{1} \boldsymbol{v}_{1}+\ldots+a_{18} \boldsymbol{v}_{18}$. The third order tensors $\boldsymbol{a}$ and $\boldsymbol{b}$ can then be replaced by two vectors of dimension 18 having respectively the components $a_{I}$ and $b_{I}$ for $I=1 . .18$. In the linear relation $\boldsymbol{b}=\mathbb{A} \odot_{3} \boldsymbol{a}$, the components of $\boldsymbol{b}$ depend on the ones of $\boldsymbol{a}$ by: $b_{I}=A_{I J} a_{J}$ where $A_{I J}$ is a matrix of dimension $18 \times 18$ which depends on 324 independent coefficients. When tensor $\mathbb{A}$ has the major symmetry, matrix $A_{I J}$ is symmetric and depends on 171 independent coefficients. Tensors $\boldsymbol{v}_{I} \otimes \boldsymbol{v}_{J}$ for $I, J=1 . .18$ constitute then an irreducible basis for general sixth order tensors. When $\mathbb{A}$ is invariant by rotation or reflection (cf. relation (2)), the total number of independent coefficients of $\mathbb{A}$ can be strongly reduced and the definition of a new irreducible basis is required. 
By adopting the above contracted notations, the transformation rule (2) reads:

$$
A_{I J}=R_{I P} R_{J Q} A_{P Q}
$$

where matrix $R_{I J}$ are the components of tensor $\mathbb{R}$ defined by $R_{i j k p q r}=$ $\left(R_{i p} R_{j q}+R_{i q} R_{j p}\right) R_{k r} / 2$ with the above contracted notations. In the case of a plane symmetry with respect to the plane $O x_{1} x_{2}, R_{I J}$ is diagonal and is given by:

$$
R_{I J}=\operatorname{diag}(\underbrace{1,1, . ., 1}_{10 \text { times }}, \underbrace{-1,-1, . .,-1}_{8 \text { times }}) .
$$

In the case of a rotation of an angle $\theta$ around the axis $O x_{3}$, the matrix $R_{I J}$ is obtained by the concatenation of different matrices as follows:

$$
R_{I J}=\left[\begin{array}{ccc}
\mathcal{R}_{i j}^{1} & \mathcal{R}_{i j}^{2} & 0 \\
-\mathcal{R}_{i j}^{2} & \mathcal{R}_{i j}^{1} & 0 \\
0 & 0 & \mathcal{R}_{i j}^{3}
\end{array}\right]
$$

In the above expressions, matrices $\mathcal{R}_{i j}^{1}, \mathcal{R}_{i j}^{2}$ are squared matrices of dimension 5 , defined by:

$$
\begin{gathered}
\mathcal{R}_{i j}^{1}=p\left[\begin{array}{ccccc}
p^{2} & q^{2} & 0 & \sqrt{2} q^{2} & 0 \\
q^{2} & p^{2} & 0 & -\sqrt{2} q^{2} & 0 \\
0 & 0 & 1 & 0 & 0 \\
\sqrt{2} q^{2} & -\sqrt{2} q^{2} & 0 & p^{2}-q^{2} & 0 \\
0 & 0 & 0 & 0 & 1
\end{array}\right], \\
\mathcal{R}_{i j}^{2}=q\left[\begin{array}{ccccc}
q^{2} & p^{2} & 0 & \sqrt{2} p^{2} & 0 \\
p^{2} & q^{2} & 0 & -\sqrt{2} p^{2} & 0 \\
0 & 0 & 1 & 0 & 0 \\
\sqrt{2} p^{2}-\sqrt{2} p^{2} & 0 & q^{2}-p^{2} & 0 \\
0 & 0 & 0 & 0 & 1
\end{array}\right] .
\end{gathered}
$$


$\mathcal{R}_{i j}^{3}$ is squared matrix of dimension 8 ; its components are:

$$
\mathcal{R}_{i j}^{3}=\left[\begin{array}{cccccccc}
1 & 0 & 0 & 0 & 0 & 0 & 0 & 0 \\
0 & p^{2} & q^{2} & 0 & 0 & 0 & 0 & -\sqrt{2} p q \\
0 & q^{2} & p^{2} & 0 & 0 & 0 & 0 & \sqrt{2} p q \\
0 & 0 & 0 & p^{2} & q^{2} & -p q & -p q & 0 \\
0 & 0 & 0 & q^{2} & p^{2} & p q & p q & 0 \\
0 & 0 & 0 & -p q & p q & p^{2} & -q^{2} & 0 \\
0 & 0 & 0 & -p q & p q & -q^{2} & p^{2} & 0 \\
0 & \sqrt{2} p q-\sqrt{2} p q & 0 & 0 & 0 & 0 & p^{2}-q^{2}
\end{array}\right]
$$

The invariance condition (15) is now verified with expressions (16) and (17) for the matrix $R_{I J}$. These computations are elementary and lead to the following form for $A_{I J}$ :

$$
A_{I J}=\left[\begin{array}{cccc}
f_{i j} & 0 & 0 & 0 \\
0 & f_{i j} & 0 & 0 \\
0 & 0 & g_{i j} & 0 \\
0 & 0 & 0 & h_{i j}
\end{array}\right]
$$

where $f_{i j}, g_{i j}$ and $h_{i j}$ are squared matrices of dimension 5,5 and 3 respectively. The following equalities hold for the components of these matrices:

$$
\left\{\begin{array}{l}
f_{21}-f_{12}=\sqrt{2}\left(f_{14}+f_{41}\right), \quad f_{11}-f_{22}=\sqrt{2}\left(f_{24}+f_{41}\right)=\sqrt{2}\left(f_{42}+f_{14}\right), \\
2 f_{44}=f_{11}+f_{22}-f_{21}-f_{12}, \quad \sqrt{2} f_{45}=f_{15}-f_{25}, \quad \sqrt{2} f_{43}=f_{13}-f_{23}, \\
\sqrt{2} f_{54}=f_{51}-f_{52}, \quad f_{34}=f_{31}-f_{32}, \\
g_{12}=g_{13}, \quad g_{14}=g_{15}, \quad g_{21}=g_{31}, \quad g_{22}=g_{33}, \quad g_{23}=g_{32}, \quad g_{24}=g_{35}, \quad(21) \quad g_{54}, \quad g_{45}=g_{54}, \\
g_{25}=g_{34}, \quad g_{41}=g_{51}, \quad g_{42}=g_{53}, \quad g_{43}=g_{55}, \quad g_{45}, \quad h_{2} h_{32}=\sqrt{2} h_{31}=g_{24}-g_{34}, \\
h_{22}=h_{11}, \quad h_{23}=h_{13}=g_{42}-g_{43}, \quad \sqrt{2}, \quad h_{11}+h_{12}=g_{44}-g_{45} .
\end{array}\right.
$$

Matrices $f_{i j}$ and $g_{i j}$ for $i, j=1 . .5$ and $h_{i j}$ for $i, j=1 . .3$ are defined by 59 coefficients. Moreover, by considering the above 28 equalities, it follows that the total number of independent coefficients for matrix $A_{I J}$ is 31 . 


\section{An irreducible basis for transversely isotropic sixth order tensors}

In this section we propose a canonical basis for transversely isotropic sixth order tensors. The dimension of $\mathbb{A} \in E_{6}$ being 31 , an irreducible basis of such kind of tensor comprises 31 independent tensors. The method of construction uses a decomposition of a third order tensor related to the transverse isotropy.

First, we decompose the third order tensor $\boldsymbol{a}$, of components $a_{i j k}$, on the form:

$$
\begin{aligned}
a_{i j k}=w_{i j k}+u_{i j} n_{k}+n_{j} v_{i k}+n_{i} v_{j k}+n_{i} n_{j} r_{k} & \\
& +\left(n_{i} s_{j}+n_{j} s_{i}\right) n_{k}+\gamma n_{i} n_{j} n_{k}
\end{aligned}
$$

where $n_{i}$ are the components of the vector $\underline{n}$ of transverse isotropy. The inner product between $w_{i j k}, u_{i j}, v_{i j}, r_{i}, s_{i}$ with $n_{i}$ is null:

$$
\begin{aligned}
& w_{i j k} n_{k}=w_{i k j} n_{k}=w_{k i j} n_{k}=0, \\
& u_{i j} n_{j}=u_{j i} n_{j}=v_{i j} n_{j}=v_{i j} n_{i}=0, \\
& r_{i} n_{i}=s_{i} n_{i}=0 .
\end{aligned}
$$

The computation of $w_{i j k}, u_{i j}, v_{i j}, r_{i}, s_{i}$ and $\gamma$ from equations (22) and (23) leads to the following expressions:

$$
\left\{\begin{array}{l}
\gamma=a_{i j k} n_{i} n_{j} n_{k}, \\
r_{i}=\pi_{i r} a_{p q r} n_{p} n_{q}, \\
s_{i}=\pi_{i p} a_{p q r} n_{q} n_{r}, \\
u_{i j}=\pi_{i p} \pi_{j q} a_{p q r} n_{r}, \\
v_{i j}=\pi_{i q} \pi_{j r} a_{p q r} n_{p}, \\
w_{i j k}=\pi_{i p} \pi_{j q} \pi_{k r} a_{p q r}
\end{array}\right.
$$

where it is recalled that $\pi_{i j}=\delta_{i j}-n_{i} n_{j}$. Note that $u_{i j}$ is symmetric, $u_{i j}=$ $u_{j i} ; v_{i j}$ which is non-symmetric, is decomposed into a symmetric and a skew symmetric tensor:

$$
v_{i j}=v_{i j}^{s}+v_{i j}^{a}
$$

with:

$$
v_{i j}^{s}=\frac{1}{2}\left(v_{i j}+v_{j i}\right), \quad v_{i j}^{a}=\frac{1}{2}\left(v_{i j}-v_{j i}\right) .
$$


Tensors $\boldsymbol{u}$ and $\boldsymbol{v}^{s}$ are decomposed into two-dimensional spherical and deviatoric parts:

$$
u_{i j}=\bar{u}_{i j}-\pi_{i j} \alpha, \quad v_{i j}^{s}=\bar{v}_{i j}^{s}-\pi_{i j} \beta
$$

with:

$$
\alpha=\frac{1}{2} \pi_{p q} a_{p q r} n_{r}, \quad \beta=\frac{1}{2} \pi_{q r} a_{p q r} n_{p}
$$

Finally we introduce a similar decomposition for $w_{i j k}$ :

$$
w_{i j k}=\bar{w}_{i j k}+p_{i} \pi_{j k}+p_{j} \pi_{i k}+q_{k} \pi_{i j}
$$

with:

$$
p_{i}=w_{i j k} \pi_{j k} / 2-w_{j k i} \pi_{j k} / 4, \quad q_{i}=3 w_{j k i} \pi_{j k} / 4-w_{i j k} \pi_{j k} / 2
$$

where the third order tensor $\overline{\boldsymbol{w}}$ is traceless: $\bar{w}_{i j j}=\bar{w}_{j j i}=0$ and is invariant by any permutation of its indices, $\bar{w}_{i j k}=\bar{w}_{k i j}=\bar{w}_{j k i}=\bar{w}_{k j i}=\bar{w}_{i k j}=\bar{w}_{j i k}$. Let us recall that any third order tensor having the minor symmetries depends on 18 independent coefficients. Each one of vectors $p, q, \underline{r}$ and $\underline{s}$, of components $p_{i}, q_{i}, r_{i}$ and $s_{i}$, depends on 2 coefficients. The second order tensors $\overline{\boldsymbol{u}}, \overline{\boldsymbol{v}}^{s}$ and $\boldsymbol{v}^{a}$ are defined by 2,2 and 1 independent coefficient. The third order tensor $\overline{\boldsymbol{w}}$ depends on 2 independent coefficients. The total number of independent coefficients of each element of the decomposition are recalled below:

\begin{tabular}{c|c|c|c|c|c|c|c|c|c|c|c}
$\alpha$ & $\beta$ & $\gamma$ & $\underline{p}$ & $\underline{q}$ & $\underline{r}$ & $\underline{s}$ & $\overline{\boldsymbol{u}}$ & $\overline{\boldsymbol{v}}^{s}$ & $\boldsymbol{v}^{a}$ & $\overline{\boldsymbol{w}}$ & $\boldsymbol{a}$ \\
\hline 1 & 1 & 1 & 2 & 2 & 2 & 2 & 2 & 2 & 1 & 2 & 18
\end{tabular}

Table 1 Number of independent coefficients of the elements of the decomposition of the third order tensor $\boldsymbol{a}$ 
The various outer products of $\bar{w}_{i j k}, \bar{v}_{i j}^{s}, \bar{u}_{i j}, v_{i j}^{a}, t_{i}, s_{i}, x, v_{m}$ and $u_{m}$ with $\pi_{i j}$ and $n_{i}$ provide 31 third order tensors having the following components:

$$
\begin{array}{rlrl}
T_{i j k}^{1}(\boldsymbol{a}) & =\bar{w}_{i j k}, & T_{i j k}^{2}(\boldsymbol{a}) & =n_{i} v_{j k}^{a}+n_{j} v_{i k}^{a}, \\
T_{i j k}^{3}(\boldsymbol{a}) & =\bar{u}_{i j} n_{k}, & T_{i j k}^{4}(\boldsymbol{a}) & =2 \bar{v}_{i j}^{s} n_{k}, \\
T_{i j k}^{5}(\boldsymbol{a}) & =\left(n_{i} \bar{u}_{j k}+n_{j} \bar{u}_{i k}\right) / 2, & T_{i j k}^{6}(\boldsymbol{a}) & =n_{i} \bar{v}_{j k}^{s}+n_{j} \bar{v}_{i k}^{s}, \\
T_{i j k}^{7}(\boldsymbol{a}) & =\alpha \pi_{i j} n_{k}, & T_{i j k}^{8}(\boldsymbol{a}) & =2 \beta \pi_{i j} n_{k}, \\
T_{i j k}^{9}(\boldsymbol{a}) & =\gamma \pi_{i j} n_{k}, & T_{i j k}^{10}(\boldsymbol{a}) & =\alpha\left(n_{i} \pi_{j k}+n_{j} \pi_{i k}\right) / 2, \\
T_{i j k}^{11}(\boldsymbol{a}) & =\beta\left(n_{i} \pi_{j k}+n_{j} \pi_{i k}\right), & T_{i j k}^{12}(\boldsymbol{a}) & =\gamma\left(n_{i} \pi_{j k}+n_{j} \pi_{i k}\right) / 2, \\
T_{i j k}^{13}(\boldsymbol{a}) & =\alpha n_{i} n_{j} n_{k}, & & T_{i j k}^{14}(\boldsymbol{a})=2 \beta n_{i} n_{j} n_{k}, \\
T_{i j k}^{15}(\boldsymbol{a}) & =\gamma n_{i} n_{j} n_{k}, & T_{i j k}^{16}(\boldsymbol{a}) & =p_{i} \pi_{j k}+p_{j} \pi_{i k}, \\
T_{i j k}^{17}(\boldsymbol{a}) & =\left(q_{i} \pi_{j k}+q_{j} \pi_{i k}\right) / 2, & T_{i j k}^{18}(\boldsymbol{a}) & =\left(r_{i} \pi_{j k}+r_{j} \pi_{i k}\right) / 2, \\
T_{i j k}^{19}(\boldsymbol{a}) & =\left(s_{i} \pi_{j k}+s_{j} \pi_{i k}\right) / 2, & & T_{i j k}^{20}(\boldsymbol{a})=2 \pi_{i j} p_{k}, \\
T_{i j k}^{21}(\boldsymbol{a}) & =\pi_{i j} q_{k}, & T_{i j k}^{22}(\boldsymbol{a}) & =\pi_{i j} r_{k}, \\
T_{i j k}^{23}(\boldsymbol{a}) & =\pi_{i j} s_{k}, & T_{i j k}^{24}(\boldsymbol{a}) & =2 n_{i} n_{j} p_{k}, \\
T_{i j k}^{25}(\boldsymbol{a}) & =n_{i} n_{j} q_{k}, & T_{i j k}^{26}(\boldsymbol{a}) & =n_{i} n_{j} r_{k}, \\
T_{i j k}^{27}(\boldsymbol{a}) & =n_{i} n_{j} s_{k}, & T_{i j k}^{28}(\boldsymbol{a})=2\left(p_{i} n_{j}+p_{j} n_{i}\right) n_{k}, \\
T_{i j k}^{29}(\boldsymbol{a})=\left(q_{i} n_{j}+q_{j} n_{i}\right) n_{k}, & T_{i j k}^{30}(\boldsymbol{a})=\left(r_{i} n_{j}+r_{j} n_{i}\right) n_{k}, \\
T_{i j k}^{31}(\boldsymbol{a})=\left(s_{i} n_{j}+s_{j} n_{i}\right) n_{k} . &
\end{array}
$$

In relation $\boldsymbol{b}=\mathbb{A} \odot_{3} \boldsymbol{a}$, the third tensor $\boldsymbol{b}$ is a transversely isotropic and linear function of the third order tensor $\boldsymbol{a}$. The third order tensors $\boldsymbol{T}^{n}(\boldsymbol{a})$ for $n=1 . .31$ provide a complete and irreducible representation for the third order valued tensor function $\boldsymbol{b}$. This is proved in appendix A. We now introduce the sixth order tensors $\mathbb{T}_{n}$ for $n=1 . .31$ such that $\boldsymbol{T}^{n}(\boldsymbol{a})=\mathbb{T}_{n} \odot_{3} \boldsymbol{a}$. The 
components of these tensors are given below:

$$
\begin{aligned}
& {\left[T_{1}\right]_{i j k p q r}=\pi_{k r}\left(\pi_{i p} \pi_{j q}+\pi_{j p} \pi_{i q}\right) / 2-3 \pi_{i j} \pi_{p q} \pi_{k r} / 4} \\
& +\pi_{i j}\left(\pi_{p r} \pi_{k q}+\pi_{q r} \pi_{k p}\right) / 4+\pi_{p q}\left(\pi_{i r} \pi_{j k}+\pi_{j r} \pi_{i k}\right) / 4 \\
& -\pi_{i k}\left(\pi_{j p} \pi_{q r}+\pi_{j q} \pi_{p r}\right) / 4-\pi_{j k}\left(\pi_{i p} \pi_{q r}+\pi_{i q} \pi_{p r}\right) / 4, \\
& {\left[T_{2}\right]_{i j k p q r}=\left(n_{i} n_{p} \pi_{j q}+n_{i} n_{q} \pi_{j p}+n_{j} n_{p} \pi_{i q}+n_{j} n_{q} \pi_{i p}\right) \pi_{k r} / 4} \\
& -\left(\pi_{i r} n_{j}+\pi_{j r} n_{i}\right) n_{q} \pi_{k p} / 4-\left(\pi_{i r} n_{j}+\pi_{j r} n_{i}\right) n_{p} \pi_{k q} / 4, \\
& {\left[T_{3}\right]_{i j k p q r}=\left(\pi_{i p} \pi_{j q}+\pi_{i q} \pi_{j p}-\pi_{i j} \pi_{p q}\right) n_{k} n_{r} / 2 \text {, }} \\
& {\left[T_{4}\right]_{i j k p q r}=-\left(n_{p} \pi_{q r}+n_{q} \pi_{p r}\right) n_{k} \pi_{i j} / 2} \\
& +\left(\pi_{i q} \pi_{j r}+\pi_{j q} \pi_{i r}\right) n_{p} n_{k} / 2+\left(\pi_{i p} \pi_{j r}+\pi_{j p} \pi_{i r}\right) n_{q} n_{k} / 2, \\
& {\left[T_{5}\right]_{i j k p q r}=-\left(n_{i} \pi_{j k}+n_{j} \pi_{i k}\right) \pi_{p q} n_{r} / 4} \\
& +\left(\pi_{i p} \pi_{k q}+\pi_{i q} \pi_{k p}\right) n_{j} n_{r} / 4+\left(\pi_{j p} \pi_{k q}+\pi_{j q} \pi_{k p}\right) n_{i} n_{r} / 4, \\
& {\left[T_{6}\right]_{i j k p q r}=\left(n_{i} n_{p} \pi_{j q}+n_{i} n_{q} \pi_{j p}+n_{j} n_{p} \pi_{i q}+n_{j} n_{q} \pi_{i p}\right) \pi_{k r} / 4} \\
& +\left(\pi_{i r} n_{j} n_{q}+\pi_{j r} n_{i} n_{q}\right) \pi_{k p} / 4+\left(\pi_{i r} n_{j} n_{p}+\pi_{j r} n_{i} n_{p}\right) \pi_{k q} / 4 \\
& -\left(n_{i} n_{p} \pi_{q r}+n_{i} n_{q} \pi_{p r}\right) \pi_{j k} / 4-\left(n_{j} n_{p} \pi_{q r}+n_{j} n_{q} \pi_{p r}\right) \pi_{i k} / 4 \\
& {\left[T_{7}\right]_{i j k p q r}=\pi_{i j} \pi_{p q} n_{k} n_{r} / 2 \text {, }} \\
& {\left[T_{8}\right]_{i j k p q r}=\left(n_{p} \pi_{q r}+n_{q} \pi_{p r}\right) n_{k} \pi_{i j} / 2 \text {, }} \\
& {\left[T_{9}\right]_{i j k p q r}=\pi_{i j} n_{p} n_{q} n_{k} n_{r},} \\
& {\left[T_{10}\right]_{i j k p q r}=\left(n_{i} \pi_{j k}+n_{j} \pi_{i k}\right) n_{r} \pi_{p q} / 4,} \\
& {\left[T_{11}\right]_{i j k p q r}=\left(n_{p} \pi_{q r}+n_{q} \pi_{p r}\right)\left(n_{i} \pi_{j k}+n_{j} \pi_{i k}\right) / 4,} \\
& {\left[T_{12}\right]_{i j k p q r}=\left(\pi_{i k} n_{j}+\pi_{j k} n_{i}\right) n_{r} n_{p} n_{q} / 2,} \\
& {\left[T_{13}\right]_{i j k p q r}=n_{i} n_{j} \pi_{p q} n_{k} n_{r} / 2 \text {, }} \\
& {\left[T_{14}\right]_{i j k p q r}=\left(\pi_{p r} n_{q}+\pi_{q r} n_{p}\right) n_{i} n_{j} n_{k} / 2,} \\
& {\left[T_{15}\right]_{i j k p q r}=n_{i} n_{j} n_{k} n_{p} n_{q} n_{r},} \\
& {\left[T_{16}\right]_{i j k p q r}=\pi_{j k}\left(\pi_{i p} \pi_{q r}+\pi_{i q} \pi_{p r}-\pi_{p q} \pi_{i r}\right) / 4} \\
& +\pi_{i k}\left(\pi_{j p} \pi_{q r}+\pi_{j q} \pi_{p r}-\pi_{p q} \pi_{j r}\right) / 4,
\end{aligned}
$$




$$
\begin{aligned}
& {\left[T_{17}\right]_{i j k p q r}=\pi_{j k}\left(3 \pi_{p q} \pi_{i r}-\pi_{i p} \pi_{q r}-\pi_{i q} \pi_{p r}\right) / 8} \\
& +\pi_{i k}\left(3 \pi_{p q} \pi_{j r}-\pi_{j p} \pi_{q r}-\pi_{j q} \pi_{p r}\right) / 8, \\
& {\left[T_{18}\right]_{i j k p q r}=\left(\pi_{i r} \pi_{j k}+\pi_{j r} \pi_{i k}\right) n_{p} n_{q} / 2 \text {, }} \\
& {\left[T_{19}\right]_{i j k p q r}=\left(\pi_{i p} n_{q}+\pi_{i q} n_{p}\right) \pi_{j k} n_{r} / 4+\left(\pi_{j p} n_{q}+\pi_{j q} n_{p}\right) \pi_{i k} n_{r} / 4 \text {, }} \\
& {\left[T_{20}\right]_{i j k p q r}=\pi_{i j}\left(\pi_{p r} \pi_{k q}+\pi_{q r} \pi_{k p}-\pi_{p q} \pi_{k r}\right) / 2 \text {, }} \\
& {\left[T_{21}\right]_{i j k p q r}=\pi_{i j}\left(3 \pi_{p q} \pi_{k r}-\pi_{p r} \pi_{k q}-\pi_{q r} \pi_{k p}\right) / 4 \text {, }} \\
& {\left[T_{22}\right]_{i j k p q r}=\pi_{i j} n_{p} n_{q} \pi_{k r},} \\
& {\left[T_{23}\right]_{i j k p q r}=\left(n_{p} \pi_{q k}+n_{q} \pi_{p k}\right) n_{r} \pi_{i j} / 2,} \\
& {\left[T_{24}\right]_{i j k p q r}=\left(\pi_{p r} \pi_{q k}+\pi_{q r} \pi_{p k}-\pi_{p q} \pi_{k r}\right) n_{i} n_{j} / 2 \text {, }} \\
& {\left[T_{25}\right]_{i j k p q r}=\left(3 \pi_{p q} \pi_{k r}-\pi_{p r} \pi_{q k}-\pi_{q r} \pi_{p k}\right) n_{i} n_{j} / 4 \text {, }} \\
& {\left[T_{26}\right]_{i j k p q r}=n_{i} n_{j} n_{p} n_{q} \pi_{k r} \text {, }} \\
& {\left[T_{27}\right]_{i j k p q r}=\left(\pi_{p k} n_{q}+\pi_{q k} n_{p}\right) n_{i} n_{j} n_{r} / 2 \text {, }} \\
& {\left[T_{28}\right]_{i j k p q r}=\left(\pi_{i p} \pi_{q r}+\pi_{i q} \pi_{p r}\right) n_{j} n_{k} / 2+\left(\pi_{j p} \pi_{q r}+\pi_{j q} \pi_{p r}\right) n_{i} n_{k} / 2} \\
& -\left(n_{i} \pi_{j r}+n_{j} \pi_{i r}\right) n_{k} \pi_{p q} / 2, \\
& {\left[T_{29}\right]_{i j k p q r}=3\left(n_{i} \pi_{j r}+n_{j} \pi_{i r}\right) n_{k} \pi_{p q} / 4-\left(\pi_{i p} \pi_{q r}+\pi_{i q} \pi_{p r}\right) n_{j} n_{k} / 4} \\
& -\left(\pi_{j p} \pi_{q r}+\pi_{j q} \pi_{p r}\right) n_{i} n_{k} / 4, \\
& {\left[T_{30}\right]_{i j k p q r}=\left(\pi_{i r} n_{j}+\pi_{j r} n_{i}\right) n_{k} n_{p} n_{q},} \\
& {\left[T_{31}\right]_{i j k p q r}=\left(\pi_{i p} n_{j} n_{q}+\pi_{i q} n_{j} n_{p}+\pi_{j p} n_{i} n_{q}+\pi_{j q} n_{i} n_{p}\right) n_{k} n_{r} / 2 .}
\end{aligned}
$$

The set of tensors $\mathbb{T}_{n}$ for $n=1 \ldots 31$ constitutes then an irreducible basis for any transversely isotropic sixth order tensor having the minor symmetries. Then any tensor $\mathbb{A} \in E_{6}$ can be read:

$$
\mathbb{A}=\sum_{n=1}^{n=31} a_{n} \mathbb{T}_{n}
$$

where the $a_{n}$ are the components of tensor $\mathbb{A}$ in the basis $\left(\mathbb{T}_{1}, \ldots, \mathbb{T}_{31}\right)$. The relations giving the coefficients $a_{n}$ for $n=1 . .31$ as functions of the components $A_{i j k p q r}$ are given in appendix B. It must be emphasized that relations (60) can also be used to prove that tensors $\mathbb{T}_{n}$ for $n=1 . .31$ are linearly independent. Indeed, in (34), tensor $\mathbb{A}$ if the components $A_{i j k p q r}$ are null and, from relations $(60)$, if $a_{n}=0$ for $n=1 . .31$.

The triple contraction between two elements of the basis $\mathbb{T}_{n}$ are given in table 2 together with tables 3,4 and 5. It can be observed that the set of tensors 
$\left(\mathbb{T}_{1}, \ldots, \mathbb{T}_{31}\right)$ is constituted of 5 groups which are independent for the composition $\odot_{3}$. These groups are $\left\{\mathbb{T}_{1}\right\},\left\{\mathbb{T}_{2}\right\},\left\{\mathbb{T}_{3}, \ldots, \mathbb{T}_{6}\right\},\left\{\mathbb{T}_{7}, \ldots, \mathbb{T}_{15}\right\},\left\{\mathbb{T}_{16}, \ldots, \mathbb{T}_{31}\right\}$, and define sub-spaces of $E_{6}$ whose dimensions are respectively: 1, 1, 4, 9, 25. This irreducible basis is constituted of two kinds of elements:

- the first kind of tensors are idempotent since they are invariants by the inner product " $\odot_{3}$ ", i.e. $\mathbb{T}_{n} \odot_{3} \mathbb{T}_{n}=\mathbb{T}_{n}$. These tensors are $\mathbb{T}_{1}, \mathbb{T}_{2}, \mathbb{T}_{3}, \mathbb{T}_{6}$, $\mathbb{T}_{7}, \mathbb{T}_{11}, \mathbb{T}_{15}, \mathbb{T}_{16}, \mathbb{T}_{21}, \mathbb{T}_{26}, \mathbb{T}_{31}$ and correspond to the tensors that appear in the diagonal of table 1 .

- other tensors are nilpotent; the inner product " $\odot_{3}$ " of these tensors by themselves gives the null tensor, ie. $\mathbb{T}_{n} \odot_{3} \mathbb{T}_{n}=0$. These tensors appear only out of the diagonal in table 1 .

It can be noticed that the irreducible basis for fourth order tensors, defined in (8), is also constituted of idempotent tensors $\left(\mathbb{T}_{1}, \mathbb{T}_{2}, \mathbb{T}_{3}\right.$ and $\left.\mathbb{T}_{6}\right)$ and nilpotent tensors (they are $\mathbb{T}_{4}$ and $\mathbb{T}_{5}$ ). Note also that similar structures has been also obtained for sixth and eighth order isotropic tensors in Monchiet and Bonnet [15], [16].

It can be observed that the set $\left(E_{6}, \odot_{3}, \mathbb{I}\right)$ defines a monoid (an algebraic structure with a single associative binary operation and an identity element). $\mathbb{I}$ represents the identity for isotropic sixth order tensors having the minor symmetries and is such that $\mathbb{I} \odot_{3} \boldsymbol{a}=\boldsymbol{a}$ for any third order tensor $\boldsymbol{a}$. The components of the sixth order identity tensor are $I_{i j k p q r}=I_{i j p q} \delta_{k r}$ where $I_{i j p q}=\left(\delta_{i p} \delta_{j q}+\delta_{i q} \delta_{j p}\right) / 2$ are the components of the identity tensor for symmetric fourth order tensors. The decomposition of $\mathbb{I}$ along the basis $\left(\mathbb{T}_{1}, \ldots, \mathbb{T}_{31}\right)$ is $\mathbb{I}=\mathbb{T}_{1}+\mathbb{T}_{2}+\mathbb{T}_{3}+\mathbb{T}_{6}+\mathbb{T}_{7}+\mathbb{T}_{11}+\mathbb{T}_{15}+\mathbb{T}_{16}+\mathbb{T}_{21}+\mathbb{T}_{26}+\mathbb{T}_{31}$ and corresponds to the sum of all idempotent tensors, which is also a property shared by isotropic tensors. As a consequence, every third order tensor can be decomposed into:

$$
\begin{aligned}
a=T^{1}(a)+ & T^{2}(a)+T^{3}(a)+T^{6}(a)+T^{7}(a)+T^{11}(a)+T^{15}(a) \\
& +T^{16}(a)+T^{21}(a)+T^{26}(a)+T^{31}(a) .
\end{aligned}
$$




\begin{tabular}{|c|c|c|ccc|ccc|ccc|}
\hline$\odot_{3}$ & $\mathbb{T}_{1}$ & $\mathbb{T}_{2}$ & $\mathbb{T}_{3}$ & $\ldots$ & $\mathbb{T}_{6}$ & $\mathbb{T}_{7}$ & $\ldots$ & $\mathbb{T}_{15}$ & $\mathbb{T}_{16}$ & $\ldots$ & $\mathbb{T}_{31}$ \\
\hline $\mathbb{T}_{1}$ & $\mathbb{T}_{1}$ & 0 & 0 & $\ldots$ & 0 & 0 & $\ldots$ & 0 & 0 & $\ldots$ & 0 \\
\hline $\mathbb{T}_{2}$ & 0 & $\mathbb{T}_{2}$ & 0 & $\ldots$ & 0 & 0 & $\ldots$ & 0 & 0 & $\ldots$ & 0 \\
\hline $\mathbb{T}_{3}$ & 0 & 0 & & & & 0 & $\ldots$ & 0 & 0 & $\ldots$ & 0 \\
$\vdots$ & $\vdots$ & $\vdots$ & & Table 3 & & $\vdots$ & $\ddots$ & $\vdots$ & $\vdots$ & $\ddots$ & $\vdots$ \\
$\mathbb{T}_{6}$ & 0 & 0 & & & & 0 & $\ldots$ & 0 & 0 & $\ldots$ & 0 \\
\hline $\mathbb{T}_{7}$ & 0 & 0 & 0 & $\ldots$ & 0 & & & & 0 & $\ldots$ & 0 \\
$\ldots$ & $\vdots$ & $\vdots$ & $\vdots$ & $\ddots$ & $\vdots$ & & Table 4 & & $\vdots$ & $\ddots$ & $\vdots$ \\
$\mathbb{T}_{15}$ & 0 & 0 & 0 & $\ldots$ & 0 & & & & 0 & $\ldots$ & 0 \\
\hline $\mathbb{T}_{16}$ & 0 & 0 & 0 & $\ldots$ & 0 & 0 & $\ldots$ & 0 & & & \\
$\ldots$ & $\vdots$ & $\vdots$ & $\vdots$ & $\ddots$ & $\vdots$ & $\vdots$ & $\ddots$ & $\vdots$ & & Table 5 & \\
$\mathbb{T}_{31}$ & 0 & 0 & 0 & $\ldots$ & 0 & 0 & $\ldots$ & 0 & & & \\
\hline
\end{tabular}

Table 2: The triple contraction between the $\mathbb{T}_{n}$ for $n=1 . .31$

\begin{tabular}{|c|c|c|c|c|}
\hline$\odot_{3}$ & $\mathbb{T}_{3}$ & $\mathbb{T}_{4}$ & $\mathbb{T}_{5}$ & $\mathbb{T}_{6}$ \\
\hline $\mathbb{T}_{3}$ & $\mathbb{T}_{3}$ & $\mathbb{T}_{4}$ & 0 & 0 \\
\hline $\mathbb{T}_{4}$ & 0 & 0 & $\mathbb{T}_{3}$ & $\mathbb{T}_{4}$ \\
\hline $\mathbb{T}_{5}$ & $\mathbb{T}_{5}$ & $\mathbb{T}_{6}$ & 0 & 0 \\
\hline $\mathbb{T}_{6}$ & 0 & 0 & $\mathbb{T}_{5}$ & $\mathbb{T}_{6}$ \\
\hline
\end{tabular}

Table 3: The triple contraction between the $\mathbb{T}_{n}$ for $n=3 . .6$ 


\begin{tabular}{|c|c|c|c|c|c|c|c|c|c|}
\hline$\odot_{3}$ & $\mathbb{T}_{7}$ & $\mathbb{T}_{8}$ & $\mathbb{T}_{9}$ & $\mathbb{T}_{10}$ & $\mathbb{T}_{11}$ & $\mathbb{T}_{12}$ & $\mathbb{T}_{13}$ & $\mathbb{T}_{14}$ & $\mathbb{T}_{15}$ \\
\hline $\mathbb{T}_{7}$ & $\mathbb{T}_{7}$ & $\mathbb{T}_{8}$ & $\mathbb{T}_{9}$ & 0 & 0 & 0 & 0 & 0 & 0 \\
\hline $\mathbb{T}_{8}$ & 0 & 0 & 0 & $\mathbb{T}_{7}$ & $\mathbb{T}_{8}$ & $\mathbb{T}_{9}$ & 0 & 0 & 0 \\
\hline $\mathbb{T}_{9}$ & 0 & 0 & 0 & 0 & 0 & 0 & $\mathbb{T}_{7}$ & $\mathbb{T}_{8}$ & $\mathbb{T}_{9}$ \\
\hline $\mathbb{T}_{10}$ & $\mathbb{T}_{10}$ & $\mathbb{T}_{11}$ & $\mathbb{T}_{12}$ & 0 & 0 & 0 & 0 & 0 & 0 \\
\hline $\mathbb{T}_{11}$ & 0 & 0 & 0 & $\mathbb{T}_{10}$ & $\mathbb{T}_{11}$ & $\mathbb{T}_{12}$ & 0 & 0 & 0 \\
\hline $\mathbb{T}_{12}$ & 0 & 0 & 0 & 0 & 0 & 0 & $\mathbb{T}_{10}$ & $\mathbb{T}_{11}$ & $\mathbb{T}_{12}$ \\
\hline $\mathbb{T}_{13}$ & $\mathbb{T}_{13}$ & $\mathbb{T}_{14}$ & $\mathbb{T}_{15}$ & 0 & 0 & 0 & 0 & 0 & 0 \\
\hline $\mathbb{T}_{14}$ & 0 & 0 & 0 & $\mathbb{T}_{13}$ & $\mathbb{T}_{14}$ & $\mathbb{T}_{15}$ & 0 & 0 & 0 \\
\hline $\mathbb{T}_{15}$ & 0 & 0 & 0 & 0 & 0 & 0 & $\mathbb{T}_{13}$ & $\mathbb{T}_{14}$ & $\mathbb{T}_{15}$ \\
\hline
\end{tabular}

Table 4: The triple contraction between the $\mathbb{T}_{n}$ for $n=7 . .15$

\begin{tabular}{|c|c|c|c|c|c|c|c|c|c|c|c|c|c|c|c|c|}
\hline$\odot_{3}$ & $\mathbb{T}_{16}$ & $\mathbb{T}_{17}$ & $\mathbb{T}_{18}$ & $\mathbb{T}_{19}$ & $\mathbb{T}_{20}$ & $\mathbb{T}_{21}$ & $\mathbb{T}_{22}$ & $\mathbb{T}_{23}$ & $\mathbb{T}_{24}$ & $\mathbb{T}_{25}$ & $\mathbb{T}_{26}$ & $\mathbb{T}_{27}$ & $\mathbb{T}_{28}$ & $\mathbb{T}_{29}$ & $\mathbb{T}_{30}$ & $\mathbb{T}_{31}$ \\
\hline $\mathbb{T}_{16}$ & $\mathbb{T}_{16}$ & $\mathbb{T}_{17}$ & $\mathbb{T}_{18}$ & $\mathbb{T}_{19}$ & 0 & 0 & 0 & 0 & 0 & 0 & 0 & 0 & 0 & 0 & 0 & 0 \\
\hline $\mathbb{T}_{17}$ & 0 & 0 & 0 & 0 & $\mathbb{T}_{16}$ & $\mathbb{T}_{17}$ & $\mathbb{T}_{18}$ & $\mathbb{T}_{19}$ & 0 & 0 & 0 & 0 & 0 & 0 & 0 & 0 \\
\hline $\mathbb{T}_{18}$ & 0 & 0 & 0 & 0 & 0 & 0 & 0 & 0 & $\mathbb{T}_{16}$ & $\mathbb{T}_{17}$ & $\mathbb{T}_{18}$ & $\mathbb{T}_{19}$ & 0 & 0 & 0 & 0 \\
\hline $\mathbb{T}_{19}$ & 0 & 0 & 0 & 0 & 0 & 0 & 0 & 0 & 0 & 0 & 0 & 0 & $\mathbb{T}_{16}$ & $\mathbb{T}_{17}$ & $\mathbb{T}_{18}$ & $\mathbb{T}_{19}$ \\
\hline $\mathbb{T}_{20}$ & $\mathbb{T}_{20}$ & $\mathbb{T}_{21}$ & $\mathbb{T}_{22}$ & $\mathbb{T}_{23}$ & 0 & 0 & 0 & 0 & 0 & 0 & 0 & 0 & 0 & 0 & 0 & 0 \\
\hline $\mathbb{T}_{21}$ & 0 & 0 & 0 & 0 & $\mathbb{T}_{20}$ & $\mathbb{T}_{21}$ & $\mathbb{T}_{22}$ & $\mathbb{T}_{23}$ & 0 & 0 & 0 & 0 & 0 & 0 & 0 & 0 \\
\hline $\mathbb{T}_{22}$ & 0 & 0 & 0 & 0 & 0 & 0 & 0 & 0 & $\mathbb{T}_{20}$ & $\mathbb{T}_{21}$ & $\mathbb{T}_{22}$ & $\mathbb{T}_{23}$ & 0 & 0 & 0 & 0 \\
\hline $\mathbb{T}_{23}$ & 0 & 0 & 0 & 0 & 0 & 0 & 0 & 0 & 0 & 0 & 0 & 0 & $\mathbb{T}_{20}$ & $\mathbb{T}_{21}$ & $\mathbb{T}_{22}$ & $\mathbb{T}_{23}$ \\
\hline $\mathbb{T}_{24}$ & $\mathbb{T}_{24}$ & $\mathbb{T}_{25}$ & $\mathbb{T}_{26}$ & $\mathbb{T}_{27}$ & 0 & 0 & 0 & 0 & 0 & 0 & 0 & 0 & 0 & 0 & 0 & 0 \\
\hline $\mathbb{T}_{25}$ & 0 & 0 & 0 & 0 & $\mathbb{T}_{24}$ & $\mathbb{T}_{25}$ & $\mathbb{T}_{26}$ & $\mathbb{T}_{27}$ & 0 & 0 & 0 & 0 & 0 & 0 & 0 & 0 \\
\hline $\mathbb{T}_{26}$ & 0 & 0 & 0 & 0 & 0 & 0 & 0 & 0 & $\mathbb{T}_{24}$ & $\mathbb{T}_{25}$ & $\mathbb{T}_{26}$ & $\mathbb{T}_{27}$ & 0 & 0 & 0 & 0 \\
\hline $\mathbb{T}_{27}$ & 0 & 0 & 0 & 0 & 0 & 0 & 0 & 0 & 0 & 0 & 0 & 0 & $\mathbb{T}_{24}$ & $\mathbb{T}_{25}$ & $\mathbb{T}_{26}$ & $\mathbb{T}_{27}$ \\
\hline $\mathbb{T}_{28}$ & $\mathbb{T}_{28}$ & $\mathbb{T}_{29}$ & $\mathbb{T}_{30}$ & $\mathbb{T}_{31}$ & 0 & 0 & 0 & 0 & 0 & 0 & 0 & 0 & 0 & 0 & 0 & 0 \\
\hline $\mathbb{T}_{29}$ & 0 & 0 & 0 & 0 & $\mathbb{T}_{28}$ & $\mathbb{T}_{29}$ & $\mathbb{T}_{30}$ & $\mathbb{T}_{31}$ & 0 & 0 & 0 & 0 & 0 & 0 & 0 & 0 \\
\hline $\mathbb{T}_{30}$ & 0 & 0 & 0 & 0 & 0 & 0 & 0 & 0 & $\mathbb{T}_{28}$ & $\mathbb{T}_{29}$ & $\mathbb{T}_{30}$ & $\mathbb{T}_{31}$ & 0 & 0 & 0 & 0 \\
\hline $\mathbb{T}_{31}$ & 0 & 0 & 0 & 0 & 0 & 0 & 0 & 0 & 0 & 0 & 0 & 0 & $\mathbb{T}_{28}$ & $\mathbb{T}_{29}$ & $\mathbb{T}_{30}$ & $\mathbb{T}_{31}$ \\
\hline
\end{tabular}


Table 5: The triple contraction between the $\mathbb{T}_{n}$ for $n=16 . .31$

By using the decomposition (35) for the third order tensor $\boldsymbol{b}=\mathbb{A} \odot_{3} \boldsymbol{a}$, we obtain:

$$
\left\{\begin{array}{l}
\boldsymbol{T}^{1}(\boldsymbol{b})=a_{1} \boldsymbol{T}^{1}(\boldsymbol{a}), \\
\boldsymbol{T}^{2}(\boldsymbol{b})=a_{2} \boldsymbol{T}^{2}(\boldsymbol{a}), \\
\boldsymbol{T}^{3}(\boldsymbol{b})=a_{3} \boldsymbol{T}^{3}(\boldsymbol{a})+a_{4} \boldsymbol{T}^{4}(\boldsymbol{a}), \\
\boldsymbol{T}^{6}(\boldsymbol{b})=a_{5} \boldsymbol{T}^{5}(\boldsymbol{a})+a_{6} \boldsymbol{T}^{6}(\boldsymbol{a}), \\
\boldsymbol{T}^{7}(\boldsymbol{b})=a_{7} \boldsymbol{T}^{7}(\boldsymbol{a})+a_{8} \boldsymbol{T}^{8}(\boldsymbol{a})+a_{9} \boldsymbol{T}^{9}(\boldsymbol{a}), \\
\boldsymbol{T}^{11}(\boldsymbol{b})=a_{10} \boldsymbol{T}^{10}(\boldsymbol{a})+a_{11} \boldsymbol{T}^{11}(\boldsymbol{a})+a_{12} \boldsymbol{T}^{12}(\boldsymbol{a}), \\
\boldsymbol{T}^{15}(\boldsymbol{b})=a_{13} \boldsymbol{T}^{13}(\boldsymbol{a})+a_{14} \boldsymbol{T}^{14}(\boldsymbol{a})+a_{15} \boldsymbol{T}^{15}(\boldsymbol{a}), \\
\boldsymbol{T}^{16}(\boldsymbol{b})=a_{16} \boldsymbol{T}^{16}(\boldsymbol{a})+a_{17} \boldsymbol{T}^{17}(\boldsymbol{a})+a_{18} \boldsymbol{T}^{18}(\boldsymbol{a})+a_{19} \boldsymbol{T}^{19}(\boldsymbol{a}), \\
\boldsymbol{T}^{21}(\boldsymbol{b})=a_{20} \boldsymbol{T}^{20}(\boldsymbol{a})+a_{21} \boldsymbol{T}^{21}(\boldsymbol{a})+a_{22} \boldsymbol{T}^{22}(\boldsymbol{a})+a_{23} \boldsymbol{T}^{23}(\boldsymbol{a}), \\
\boldsymbol{T}^{26}(\boldsymbol{b})=a_{24} \boldsymbol{T}^{24}(\boldsymbol{a})+a_{25} \boldsymbol{T}^{25}(\boldsymbol{a})+a_{26} \boldsymbol{T}^{26}(\boldsymbol{a})+a_{27} \boldsymbol{T}^{27}(\boldsymbol{a}), \\
\boldsymbol{T}^{31}(\boldsymbol{b})=a_{28} \boldsymbol{T}^{28}(\boldsymbol{a})+a_{29} \boldsymbol{T}^{29}(\boldsymbol{a})+a_{30} \boldsymbol{T}^{30}(\boldsymbol{a})+a_{31} \boldsymbol{T}^{31}(\boldsymbol{a})
\end{array}\right.
$$

where coefficients $a_{n}$ for $n=1 . .31$ are the components of $\mathbb{A}$ as defined in (34). Consequently, it is possible to represent $\mathbb{A}$ by two scalars, and three matrices of dimension 2, 3 and 4 with the following symbolic notations:

$$
\mathbb{A}=\left\{a_{1}, a_{2}, A_{1}, A_{2}, A_{3}\right\} .
$$

The components of $A_{1}, A_{2}$ and $A_{3}$ are:

$$
A_{1}=\left[\begin{array}{ll}
a_{3} & a_{4} \\
a_{5} & a_{6}
\end{array}\right], A_{2}=\left[\begin{array}{ccc}
a_{7} & a_{8} & a_{9} \\
a_{10} & a_{11} & a_{12} \\
a_{13} & a_{14} & a_{15}
\end{array}\right], A_{3}=\left[\begin{array}{llll}
a_{16} & a_{17} & a_{18} & a_{19} \\
a_{20} & a_{21} & a_{22} & a_{23} \\
a_{24} & a_{25} & a_{26} & a_{27} \\
a_{28} & a_{29} & a_{30} & a_{31}
\end{array}\right]
$$

and the $a_{n}$ for $n=1 . .31$ are the components of $\mathbb{A}$ in the basis $\mathbb{T}_{n}$ with $n=1 . .31$ as defined in relation (34). For instance the identity $\mathbb{I}$ reads:

$$
\mathbb{I}=\left\{1,1,\left[\begin{array}{ll}
1 & 0 \\
0 & 1
\end{array}\right],\left[\begin{array}{lll}
1 & 0 & 0 \\
0 & 1 & 0 \\
0 & 0 & 1
\end{array}\right],\left[\begin{array}{llll}
1 & 0 & 0 & 0 \\
0 & 1 & 0 & 0 \\
0 & 0 & 1 & 0 \\
0 & 0 & 0 & 1
\end{array}\right]\right\} \text {. }
$$


Let us now introduce a second tensor $\mathbb{B}$, whose components in the basis $\left(\mathbb{T}_{1}, \ldots, \mathbb{T}_{31}\right)$ are denoted by $b_{n}$. We use the same compact representation for this tensor: $\mathbb{B}=\left\{b_{1}, b_{2}, B_{1}, B_{2}, B_{3}\right\}$. We now introduce the tensor $\mathbb{C}$ defined by $\mathbb{C}=\mathbb{A} \odot_{3} \mathbb{B} ;$ its components along $\left(\mathbb{T}_{1}, \ldots, \mathbb{T}_{31}\right)$ are denoted by $c_{n}$ for $n=1 . .31$. The elements of the compact representation of tensor $\mathbb{C}$ are $\left\{c_{1}, c_{2}, C_{1}, C_{2}, C_{3}\right\}$ with:

$$
c_{1}=a_{1} b_{1}, \quad c_{2}=a_{2} b_{2}, \quad C_{1}=A_{1} B_{1}, \quad C_{2}=A_{2} B_{2}, \quad C_{3}=A_{3} B_{3}
$$

where the standard matrix product rule is applied between the matrices $A_{n}$ and $B_{n}$ for obtaining the matrix $C_{n}$. The inversion of tensor $\mathbb{A}$ is then obviously obtained by inverting all the elements of its compact representation:

$$
\mathbb{A}^{-1}=\left\{\frac{1}{a_{1}}, \frac{1}{a_{2}}, A_{1}^{-1}, A_{2}^{-1}, A_{3}^{-1}\right\} .
$$

The existence of such an inverse is obviously dependent on the inverse of its components. As an illustration purpose, we now aim at applying this formalism for deriving closed-form solutions of the second order inhomogeneity problem.

\section{Application to the second order inhomogenity problem}

Eshelby's solutions $[5,6]$ for inclusions and for equivalent inhomogeneity problems are fundamental to many problems in material science, mechanics of composite, etc. In the terminology of Eshelby [5] and Mura [19], an inclusion denotes a subdomain subjected to an eigenstrain while an inhomogeneity is a domain whose elastic properties differ from those of the surrounding medium. Eshelby's results $[5,6]$ are well known for the case of a prescribed constant eigenstrain in an infinite isotropic medium: it gives a constant strain field inside an ellipsoidal inclusion while the exterior point solution (outside the inclusion) is heterogeneous. The strain field within the inclusion is then explicitly given as a function of the eigenstrain by means of the well-known fourth order Eshelby's tensor. The Eshelby equivalent method handles the problem of a single ellipsoidal inhomogeneity by replacing it with an inclusion having properly chosen eigenstrains. The strain field within the inhomogeneity remains constant and can be expressed in terms of the remote constant strain field by inverting a fourth order tensor which does not possess the major symmetry. Later, Sendeckyj [22], Moschovidis [17], Moschovidis and Mura [18], Asaro and Barnett [1], generalize Eshelby's solution to the case of prescribed polynomial fields. In these studies, the following result has been proved: the strain in an ellipsoidal subdomain of an infinite linear elastic medium which undergoes an eigenstrain on the form of a polynomial of degree $N$, is also a polynomial with the same degree $N$. The expansions of the eigenstrain and of the interior point solution for the strain field along polynomial functions introduce tensors of order 2, 3, 4 etc and higher order Eshelby tensors of order 6, 8 etc. It has been pointed out in Mura [19] that the strain disturbance due to a polynomial type remote strain field of degree $N$ can be simulated by an appropriate 
polynomial eigenstrain field of degree $N$. Note that the utility of the result for $N=1$ has been addressed by Eshelby [7] for treating ellipsoidal inhomogeneities submitted to far-field torsion and flexure. Solutions to higher order inclusion problems are also the bases of numerous works dealing with multiple ellipsoidal inhomogeneities, among which Moschovidis and Mura [18], Mura [19], Shodja and Sarvestani [23], Shodja et al. [24], Benedikt et al. [2].

In the next section we propose to recall the solution of the second order inclusion problem and we deal with the problem of an ellipsoidal inhomogeneity submitted to a remote gradient of strain. Closed form solutions are provided in section 5.2 for the case of a cylindrical inhomogeneity for which the sixth order tensor, which has to be inverted, presents a transverse isotropy, the axis of isotropy being parallel to the axis of the cylinder.

\subsection{The general case of an ellipsoidal inhomogeneity}

Consider an ellipsoidal inhomogeneity, of stiffness tensor $\mathbb{C}$, embedded in an infinite elastic matrix of stiffness tensor $\mathbb{C}^{0}$. We denote by $\left(x_{1}, x_{2}, x_{3}\right)$ the cartesian coordinates, $\left(\underline{e}_{1}, \underline{e}_{2}, \underline{e}_{3}\right)$ the orthonormal basis and by $a_{1}, a_{2}, a_{3}$ the radii of the ellipsoid along the three axes of the cartesian frame. The inclusion is located at the origin and its volume is defined by:

$$
\frac{x_{1}^{2}}{a_{1}^{2}}+\frac{x_{2}^{2}}{a_{2}^{2}}+\frac{x_{3}^{2}}{a_{3}^{2}} \leq 1 .
$$

The ellipsoidal inhomogeneity contains an isotropic elastic material, $\mu$ and $\lambda$ being its Lamé coefficients. The infinite medium is also isotropic and its elastic moduli are denoted by $\mu_{0}$ and $\lambda_{0}$. This inhomogeneity is subjected to a remote strain field on the form $\varepsilon_{i j}^{\infty}=b_{i j k} x_{k}$ where $\underline{x}$, of components $x_{k}$, denotes the vector position. This problem is called "second order inhomogeneity problem" since an uniform gradient of strain is applied at the infinity instead of the constant strain considered by Eshelby [5]. The strain disturbance due to the application of $b_{i j k}$ at infinity can be recovered by considering an appropriate inclusion problem. This auxiliary problem is the following: an ellipsoidal domain, defined by (42), is subjected to a prescribed eigenstrain on the form $\varepsilon_{i j}^{*}(x)=e_{i j k} x_{k}$ (with $e_{i j k}=e_{j i k}$ ). As shown in Sendeckyj [22], Moschovidis [17], the solution of this auxiliary problem is:

$$
u_{i}(x)=\frac{1}{8 \pi\left(1-\nu_{0}\right)}\left\{\Psi_{r, p q i} e_{p q r}-2 \nu_{0} \Phi_{r, i} e_{p p r}-4\left(1-\nu_{0}\right) \Phi_{r, k} e_{i k r}\right\} .
$$

The associated strain field, $\varepsilon(x)$, reads:

$$
\begin{aligned}
\varepsilon_{i j}(x)=\frac{1}{8 \pi\left(1-\nu_{0}\right)}\left\{\Psi_{r, p q i j} e_{p q r}-2 \nu_{0} \Phi_{r, i j} e_{p p r}\right. & \\
& \left.-2\left(1-\nu_{0}\right)\left(\Phi_{r, j k} e_{i k r}+\Phi_{r, i k} e_{j k r}\right)\right\}
\end{aligned}
$$


in which $\Phi_{i}$ and $\Psi_{i}$ are respectively harmonic and bi-harmonic potentials whose expressions can be also found in the book of Mura [19] (see equations 12.13 and 12.14) and are recalled in the appendix C. The strain field (44) for an interior point (i.e. within the ellipsoidal domain) is linear according to the vector position and can be put into the form:

$$
\varepsilon_{i j}(x)=S_{i j k p q r} e_{p q r} x_{k}
$$

where $S_{i j k p q r}$ are the components of the sixth order Eshelby tensor denoted $\mathbb{S}$. The associated stress field reads:

$$
\sigma_{i j}(x)=C_{i j m n}^{0}\left(S_{m n k p q r} e_{p q r}-e_{m n k}\right) x_{k} .
$$

The components of the sixth order Eshelby tensor, $\mathbb{S}$, have not been explicitly derived in Mura [19]; their computation is detailed in appendix $\mathrm{C}$ and the final expression is given below:

$$
\begin{aligned}
& 8 \pi\left(1-\nu_{0}\right) S_{i j k p q r}=\left\{\delta_{i j} \delta_{k r} \delta_{p q}\left[\mathcal{T}_{I P R}+2 \nu_{0} \mathcal{I}_{I R}\right]\right. \\
& +2 I_{i j p q} \delta_{k r}\left[\mathcal{T}_{I J R}+\left(1-\nu_{0}\right)\left(\mathcal{I}_{I R}+\mathcal{I}_{J R}\right)\right] \\
& +2 I_{p q k r} \delta_{i j} \mathcal{T}_{I K R}+2 I_{i j k r} \delta_{p q}\left[\mathcal{T}_{I J P}+2 \nu_{0} \mathcal{I}_{I J}\right] \\
& +2\left(I_{i j p r} \delta_{k q}+\mathcal{I}_{i j q r} \delta_{k p}\right)\left[\mathcal{T}_{P Q R}+\left(1-\nu_{0}\right) \mathcal{I}_{K R}\right] \\
& \left.+2\left(I_{i j k p} \delta_{q r}+I_{i j k q} \delta_{p r}\right)\left[\mathcal{T}_{K P Q}+\left(1-\nu_{0}\right) \mathcal{I}_{K R}\right]\right\} a_{R}^{2} .
\end{aligned}
$$

The following summation convention has been used: repeated lower case indices are summed from 1 to 3 ; upper case indices (unlike in section 3) take on the same values as the corresponding lower case ones but are not summed. For example, in the monomial $a_{i} a_{i} b_{I}$, the repeated index is $i$ and the upper case index takes the same value as $i$; it gives: $a_{i} a_{i} b_{I}=a_{1}^{2} b_{1}+a_{2}^{2} b_{2}+a_{3}^{2} b_{3}$. The reader can refer to Mura [19] for more details and examples about this summation convention. The $\mathcal{I}_{i j}$ and $\mathcal{T}_{i j k}$ coefficients are defined by the following elliptic integrals:

$$
\begin{aligned}
& \mathcal{I}_{i j}=2 \pi a_{1} a_{2} a_{3} \int_{0}^{+\infty} \frac{d s}{\left(a_{i}^{2}+s\right)\left(a_{j}^{2}+s\right) \Delta(s)}, \\
& \mathcal{T}_{i j k}=2 \pi a_{1} a_{2} a_{3} \int_{0}^{+\infty} \frac{s d s}{\left(a_{i}^{2}+s\right)\left(a_{j}^{2}+s\right)\left(a_{k}^{2}+s\right) \Delta(s)}
\end{aligned}
$$

with: $\Delta(s)=\left(a_{1}^{2}+s\right)^{1 / 2}\left(a_{2}^{2}+s\right)^{1 / 2}\left(a_{3}^{2}+s\right)^{1 / 2}$. Note that $\mathcal{I}_{i j}$ and $\mathcal{T}_{i j k}$ are invariant by any permutation of their indices. The closed form expressions of the integrals appearing in (48) can be found in Moschovidis [17], Mura [19] for the case of a spheroidal inhomogeneity and particularly for the special case of a cylinder. 
Let us now come back to the inhomogeneity problem. Using the equivalent Eshelby inclusion method, the eigenstrain $\varepsilon_{i j}^{*}(x)$ is chosen as:

$$
\varepsilon_{i j}^{*}(x)=-Y_{i j p q} \varepsilon_{p q}(x)
$$

where $Y_{i j p q}$ is defined by:

$$
Y_{i j p q}=\left(1-\frac{k}{k_{0}}\right) J_{i j p q}+\left(1-\frac{\mu}{\mu_{0}}\right) K_{i j p q}
$$

where $k=\lambda+2 \mu / 3$ is the bulk modulus of the inclusion while $k_{0}=\lambda_{0}+2 \mu_{0} / 3$ is the bulk modulus of the infinite matrix. In (50), $J_{i j p q}=\delta_{i j} \delta_{p q} / 3$ and $K_{i j p q}=$ $I_{i j p q}-J_{i j p q}$ where it is recalled that $I_{i j p q}=\left(\delta_{i p} \delta_{j q}+\delta_{i q} \delta_{j p}\right) / 2$. The strain field in the ellipsoidal inhomogeneity is also linear according to the vector position and can then be written as $\varepsilon_{i j}(x)=a_{i j k} x_{k}$ where $a_{i j k}$ are the components of a constant third order tensor $\boldsymbol{a}$ which possesses the symmetry with respect to its two first indices. The strain field, solution of the inhomogeneity problem, is the sum of: (i) the prescribed remote strain field $\varepsilon_{i j}^{\infty}=b_{i j k} x_{k}$, (ii) the disturbed strain field due to the applied eigenstrain $\varepsilon_{i j}^{*}(x)=e_{i j k}$ where $e_{i j k}$ is related to $a_{i j k}$ by $e_{i j k}=S_{i j k p q r} a_{p q r}$. It follows that $\boldsymbol{a}$ is solution of a linear system on the form (1) in which the components of $\mathbb{A}$ are given by:

$$
A_{i j k p q r}=I_{i j k p q r}-S_{i j k m n r} Y_{m n p q}
$$

where it is recalled that $I_{i j k p q r}$ are the components of the sixth order identity tensor having the minor symmetries (see section 4).

\subsection{Closed-form solution for a cylindrical inhomogeneity}

We propose here to consider the case of the cylindrical inhomogeneity. We put $a_{1}=a_{2}=a$ ( $a$ being the radius of the circular section), while $a_{3}$ tends to infinity. The second order Eshelby tensor $\mathbb{S}$ defined in equation (47) with the definitions $(48)$ can be represented along the basis $\left(\mathbb{T}_{1}, \ldots, \mathbb{T}_{31}\right)$ where $\underline{e}_{3}$ is the direction of transverse isotropy. For the computation of the elements of the compact representation of $\mathbb{S}$, denoted $\left\{s_{1}, s_{2}, S_{1}, S_{2}, S_{3}\right\}$, we use the base change relations provided in appendix $\mathrm{B}$. The final expressions for the components of the Eshelby tensor are (see appendix D for the detailed expressions 
of coefficients $s_{n}$ in the case of a spheroidal inhomogeneity):

$$
\begin{aligned}
& s_{1}=\frac{13-4 \nu_{0}}{4}, s_{2}=0, \\
& S_{1}=\frac{1}{4\left(1-\nu_{0}\right)}\left[\begin{array}{ccc}
3-4 \nu_{0} & 0 \\
2\left(1-2 \nu_{0}\right) & 2\left(1-\nu_{0}\right)
\end{array}\right], \\
& S_{2}=\frac{1}{2\left(1-\nu_{0}\right)}\left[\begin{array}{ccc}
1 & 0 & 2 \nu_{0} \\
2 \nu_{0} & 2\left(1-\nu_{0}\right) & 2 \\
0 & 0 & 0
\end{array}\right], \\
& S_{3}=\frac{1}{4\left(1-\nu_{0}\right)}\left[\begin{array}{cccc}
4\left(1-\nu_{0}\right) & 2 & 2 \nu_{0} & 2\left(1-2 \nu_{0}\right) \\
0 & 1 & \nu_{0} & 1 \\
0 & 0 & 0 & 4\left(1-\nu_{0}\right) \\
0 & 0 & 0 & 2\left(1-\nu_{0}\right)
\end{array}\right] .
\end{aligned}
$$

Tensor $\mathbb{A}$, defined by (51), is also a transversely isotropic sixth order tensor. Its representation by means of the symbolic notation $\mathbb{A}=\left\{a_{1}, a_{2}, A_{1}, A_{2}, A_{3}\right\}$ is then possible. For the computation of $\mathbb{A}$, it is convenient to transform the inner product $S_{i j k m n r} Y_{m n p q}$ into a triple contraction between two sixth order tensors: tensor $\mathbb{S}$ and tensor $\mathbb{Y}^{*}$ whose components are $Y_{i j k p q r}^{*}=Y_{i j p q} \delta_{k r}$ where $Y_{i j p q}$ is defined by (50). In this manner, tensor $\mathbb{A}$ can be rewritten as $\mathbb{A}=\mathbb{I}-\mathbb{S} \odot_{3} \mathbb{Y}^{*}$ where it is recalled that $\mathbb{I}$ is the identity for sixth order tensors having minor symmetries and for the triple contraction $\odot_{3}$. The compact representation of tensor $\mathbb{Y}^{*}$ is $\left\{\xi, \xi, Y_{1}, Y_{2}, Y_{3}\right\}$ with:

$$
\begin{aligned}
& Y_{1}=\left[\begin{array}{ll}
\xi & 0 \\
0 & \xi
\end{array}\right], Y_{2}=\left[\begin{array}{ccc}
(2 \kappa+\xi) / 3 & 0 & (\kappa-\xi) / 3 \\
0 & \xi & 0 \\
2(\kappa-\xi) / 3 & 0 & (\kappa+2 \xi) / 3
\end{array}\right], \\
& Y_{3}=\left[\begin{array}{cccc}
\xi & 0 & 0 & 0 \\
(\kappa-\xi) / 3 & (2 \kappa+\xi) / 3 & (\kappa-\xi) / 3 & 0 \\
(\kappa-\xi) / 3 & 2(\kappa-\xi) / 3 & (\kappa+2 \xi) / 3 & 0 \\
0 & 0 & 0 & \xi
\end{array}\right]
\end{aligned}
$$

in which $\kappa$ and $\xi$ are given by:

$$
\kappa=1-\frac{k}{k_{0}}, \quad \xi=1-\frac{\mu}{\mu_{0}} .
$$


Finally, the inversion of $\mathbb{A}$ is performed by inverting the elements of its compact representation. It leads to:

$$
\begin{aligned}
& b_{1}=\frac{4\left(1-\nu_{0}\right)}{\Delta_{1}}, \quad b_{2}=1, \quad b_{3}=b_{1}, \quad b_{4}=0, \quad b_{5}=\frac{4 \xi\left(1-2 \nu_{0}\right)}{(2-\xi) \Delta_{1}}, \\
& b_{6}=\frac{2 \xi}{2-\xi}, \quad b_{7}=\frac{6\left(1-\nu_{0}\right)}{\Delta_{2}}, \quad b_{8}=0, \quad b_{9}=\frac{\kappa\left(1+\nu_{0}\right)-\xi\left(1-2 \nu_{0}\right)}{\Delta_{2}}, \\
& b_{10}=\frac{2 \kappa\left(1+\nu_{0}\right)-\xi\left(2-\nu_{0}\right)}{(1-\xi) \Delta_{2}}, \quad b_{11}=\frac{1}{1-\xi}, \\
& b_{12}=\frac{2 \kappa\left(1+\nu_{0}\right)+2 \xi\left(2-\nu_{0}\right)-3 \kappa \xi\left(1+\nu_{0}\right)}{(1-\xi) \Delta_{2}}, \\
& b_{13}=b_{14}=0, \quad b_{15}=1, \quad b_{16}=\frac{12\left(1-\nu_{0}\right)-2 \kappa\left(1+\nu_{0}\right)-\xi\left(1-2 \nu_{0}\right)}{\Delta_{3}}, \\
& b_{17}=2 \frac{2 \kappa\left(1+\nu_{0}\right)+\xi\left(1-2 \nu_{0}\right)}{\Delta_{3}}, \quad b_{18}=\frac{2 \kappa\left(1+\nu_{0}\right)-2 \xi\left(1-2 \nu_{0}\right)}{\Delta_{3}}, \\
& b_{19}=\frac{6 \xi\left(1-2 \nu_{0}\right)}{\Delta_{3}}, \quad b_{20}=\frac{(\kappa-\xi)\left(1+\nu_{0}\right)}{\Delta_{3}}, \\
& b_{21}=2 \frac{6\left(1-\nu_{0}\right)-\kappa\left(1+\nu_{0}\right)+\xi\left(7 \nu_{0}-5\right)}{\Delta_{3}}, \\
& b_{22}=\frac{\left(\kappa\left(1+\nu_{0}\right)-\xi\left(1-2 \nu_{0}\right)\right)(1-\xi)}{\Delta_{3}}, \quad b_{23}=\frac{3 \xi(1-\xi)\left(1-2 \nu_{0}\right)}{\Delta_{3}}-\frac{\xi}{2-\xi} \\
& b_{24}=b_{25}=0, \quad b_{26}=1, \quad b_{27}=\frac{2 \xi}{2-\xi}, \quad b_{28}=b_{29}=b_{30}=0, \quad b_{31}=\frac{2}{2-\xi}
\end{aligned}
$$

with:

$$
\begin{aligned}
& \Delta_{1}=4\left(1-\nu_{0}\right)+\xi\left(4 \nu_{0}-3\right) \\
& \Delta_{2}=6\left(1-\nu_{0}\right)-2 \kappa\left(1+\nu_{0}\right)-\xi\left(1-2 \nu_{0}\right) \\
& \Delta_{3}=12\left(1-\nu_{0}\right)-4 \kappa\left(1+\nu_{0}\right)+\xi\left(16 \nu_{0}-11\right)+2 \kappa \xi\left(1+\nu_{0}\right)+\xi^{2}\left(1-2 \nu_{0}\right)
\end{aligned}
$$

which allows the explicit computation of the strain field inside the cylinder produced by the heterogeneity problem.

\section{Conclusion}

In this paper we have provided two new results:

- The first concerns the linear algebra of transversely isotropic sixth order tensors having the minor symmetries. For this class of symmetry, it has been shown that any sixth order tensor depends on 31 independent coefficients. An irreducible basis for such tensors has then been provided as well 
as a representation by means of two scalars and three matrices of dimensions 2,3 , and 4 . This formalism is shown to be very useful for performing the classical tensorial operations since it reduces to matrix operations on matrices possessing small dimensions.

- The second result has concerned the area of micromechanics of heterogenous materials. More precisely, we have derived the strain field occurring within a cylindrical elastic inhomogeneity embedded in an infinite elastic matrix and submitted to a remote gradient of strain. The Eshelby equivalent method, which has been employed for deriving the closed-form solution, requires the inversion of a transversely isotropic sixth order tensor for which the tensorial formalism introduced in the first part of the paper has been used.

The compact representation proposed in this paper for transversely isotropic sixth order tensor could be also used for deriving various results in the context of micromechanics of heterogeneous material (for instance, the problems related to the interaction between two inhomogeneities [24]). Moreover, this tensorial formalism can be also used for deriving the condition of positiveness of elastic energy of generalized continuum theories (as already done in the case of isotropic materials in [16]).

\section{References}

1. Asaro R.J., Barnett D.M. The non-uniform transformation strain problem for an anisotropic ellipsoidal inclusion. J. Mech. Phys. Solids 23(1), 77-83 (1975).

2. Benedikt B., Lewis M., Rangaswamy P. On elastic interactions between spherical inclusions by the equivalent inclusion method. Comput. Mater. Science 37, 380-392 (2006).

3. Bona A., Bucataru I., Slawinski M.A. Coordinate-free characterization of symmetry classes of elasticity tensors. J. Elasticity 87(2-3), 109-132, 2007.

4. Chadwick P., Vianello M., Cowin S.C. A new proof that the number of linear elastic symmetries is eight. J. Mech. Phys. Solids. 49, 2471-2492 (2001).

5. Eshelby J.D. The determination of the elastic field of an ellipsoidal inclusion, and related problem. Proc. R. Soc. A 241, 376-396 (1957).

6. Eshelby J.D. The elastic field outside an ellipsoidal inclusion. Proc. Roy. Soc. London A. 252, 561-569 (1959).

7. Eshelby J.D. Progress in solid mechanics: Vol. 1. Edited by I. N. Sneddon and R. Hill, North-Holland Publishing Company, Amsterdam (1960).

8. Forte S., Vianello M. Symmetry classes for elasticity tensors. J. Elast. 43(2), 81-108 (1996).

9. Furuhashi R., Mura T. On the equivalent inclusion method and impotent eigenstrains. J. Elast. 9, 263-270 (1979).

10. Gurtin M.E. The Linear Theory of Elasticity. In Handbuch der Physik, Vol. Via/2 (ed. C.Truesdell). Springer, Berlin-Heidelberg-New York (1972).

11. Hou Y.-Z., Del Piero G. On the completeness of the crystallographic symmetries in the description of the symmetries of the elastic tensor. J. Elast. 25, 203-246 (1991).

12. Love A.E.H. A Treatise on the Mathematical Theory of Elasticity, Fourth Edition. Cambridge University Press (1927).

13. Mindlin R.D. Micro-structure in linear elasticity. Arch. Rat. Mech. Anal. 16, 51-78 (1964).

14. Mindlin R.D., Eshel N.N. On first strain-gradient theory in linear elasticity. Int. J. Solids Struct. 4, 109-124 (1968). 
15. Monchiet V., Bonnet, G. Inversion of higher order isotropic tensors with minor symmetries and solution of higher order heterogeneity problems. Proc. Roy. Soc. London A. 467(2126), 314-332, (2011)

16. Monchiet V., Bonnet, G. On the inversion of non symmetric sixth order isotropic tensors and conditions of positiveness of third order tensor valued quadratic functions. Mech. Res. Com. 38(4), 326-329 (2011).

17. Moschovidis Z.A. Two ellipsoidal inhomogeneities and related problems treated by the equivalent inclusion method, Ph.D. thesis, Northwestern University, Illinois (1975).

18. Moschovidis Z.A., Mura T. Two-ellipsoidal inhomogeneities by the equivalent inclusion method, ASME J. Appl. Mech., 42, 847 Ü852 (1975).

19. Mura T. Micromechanics of Defects in Solids. Dordrecht: Martinus Nijhoff (1987).

20. Nyashin Y., Lokhov V., Ziegler F. Decomposition method in linear elastic problems with eigenstrain. Z. Angew. Math. Mech. 85(8), 557-570 (2005).

21. Nye J.F., Physical properties of crystals. Oxford Univ. Press (1985).

22. Sendeckyj G.P. Ellipsoidal Inhomogeneity Problem. Ph.D. dissertation, Northwestern University, Evanston, IL (1967).

23. Shodja H.M., Sarvestani A.S. Elastic fields in double inhomogeneity by the equivalent inclusion method. J. Appl. Mech. 68(3), 3-10 (2001).

24. Shodja H.M., Rad I.Z., Soheilifard R. Interacting cracks and ellipsoidal inhomogeneities by the equivalent inclusion method. J. Mech. Phys. Solids. 51, 945-960 (2003).

25. Shodja H.M., Shokrolahi-Zadeh B. Ellipsoidal domains: piecewise non uniform and impotent eigenstrain fields. J. Elast. 86 1-18 (2007).

26. Suiker A.S.J., Chang, C.S. Application of higher order theory for formulating enhanced continuum models. Acta Mech. 142, 223-234 (2000).

27. Sutcliffe S. Spectral decomposition of the elasticity tensor. Trans. ASME J. Appl. Mech. 59, 762-773 (1992).

28. Thurston R.N. Waves in solids. In C. Truesdell, editor, Mechanics of Solids IV volume Via/4 of Handbuch der Physik, pp. 109-308. Springer, Berlin (1974).

29. Toupin R. Elastic materials with couple-stresses. Arch. Rat. Mech. Anal. 11 1, 385-414 (1962).

30. Voigt W. Lehrbuch der Krystallphysik. Teubner, Leipzig (1928).

31. Walpole L.J. Elastic behavior of composite materials : theoretical foundations. Advances in App. Mech., 21 : 169-243 (1981).

32. Walpole L.J. Fourth-rank tensors of the thirthy-two crystal classes: multiplication table. Proc. R. Soc. Lond. A., 391 : 149-179 (1984).

\section{A Demonstration of the completeness and irreducibility of the $T^{n}(a)$}

We aim at proving that the third order tensors $\boldsymbol{T}^{n}(\boldsymbol{a})$, defined by relations (31), are linearly independent. Consider then the following third order tensor $c$ defined by:

$$
c=\sum_{n} \varphi_{n} \boldsymbol{T}^{n}(\boldsymbol{a})
$$


Replacing in relations (36) tensor $\boldsymbol{b}$ by $\boldsymbol{c}$ and coefficients $a_{n}$ by $\varphi_{n}$, the condition $\boldsymbol{c}=0$ leads to:

$$
\left\{\begin{array}{l}
\varphi_{1} T^{1}(a)=0, \\
\varphi_{2} T^{2}(a)=0, \\
\varphi_{3} T^{3}(a)+\varphi_{4} T^{4}(a)=0, \\
\varphi_{5} T^{5}(a)+\varphi_{6} T^{6}(a)=0, \\
\varphi_{7} T^{7}(a)+\varphi_{8} T^{8}(a)+\varphi_{9} T^{9}(a)=0, \\
\varphi_{10} T^{10}(a)+\varphi_{11} T^{11}(a)+\varphi_{12} T^{12}(a)=0, \\
\varphi_{13} T^{13}(a)+\varphi_{14} T^{14}(a)+\varphi_{15} T^{15}(a)=0, \\
\varphi_{16} T^{16}(a)+\varphi_{17} T^{17}(a)+\varphi_{18} T^{18}(a)+\varphi_{19} T^{19}(a)=0, \\
\varphi_{20} T^{20}(a)+\varphi_{21} T^{21}(a)+\varphi_{22} T^{22}(a)+\varphi_{23} T^{23}(a)=0, \\
\varphi_{24} T^{24}(a)+\varphi_{25} T^{25}(a)+\varphi_{26} T^{26}(a)+\varphi_{27} T^{27}(a)=0, \\
\varphi_{28} T^{28}(a)+\varphi_{29} T^{29}(a)+\varphi_{30} T^{30}(a)+\varphi_{31} T^{31}(a)=0 .
\end{array}\right.
$$

Using the defintions for the $\boldsymbol{T}^{n}(\boldsymbol{a})$ (see relations (31)), it can be easily show that the above relations also read:

$$
\left\{\begin{array}{l}
\varphi_{1} \overline{\boldsymbol{w}}=0, \\
\varphi_{2} \boldsymbol{v}^{a}=0, \\
\varphi_{3} \overline{\boldsymbol{u}}+2 \varphi_{4} \overline{\boldsymbol{v}}^{s}=0, \\
\varphi_{5} \overline{\boldsymbol{u}}+2 \varphi_{6} \overline{\boldsymbol{v}}^{s}=0, \\
\varphi_{7} \alpha+2 \varphi_{8} \beta+\varphi_{9} \gamma=0, \\
\varphi_{10} \alpha+2 \varphi_{11} \beta+\varphi_{12} \gamma=0, \\
\varphi_{13} \alpha+2 \varphi_{14} \beta+\varphi_{15} \gamma=0, \\
2 \varphi_{16} \underline{p}+\varphi_{17} \underline{q}+\varphi_{18} \underline{r}+\varphi_{19} \underline{s}=0, \\
2 \varphi_{20} \underline{p}+\varphi_{21} \underline{q}+\varphi_{22} \underline{r}+\varphi_{23} \underline{s}=0, \\
2 \varphi_{24} \underline{p}+\varphi_{25} \underline{q}+\varphi_{26} \underline{r}+\varphi_{27} \underline{s}=0, \\
2 \varphi_{28} \underline{p}+\varphi_{29} \underline{q}+\varphi_{30} \underline{r}+\varphi_{31} \underline{s}=0 .
\end{array}\right.
$$

Quantities $\alpha, \beta, \gamma, p, q, r, s, \overline{\boldsymbol{u}}, \overline{\boldsymbol{v}}^{s}, \boldsymbol{v}^{a}, \overline{\boldsymbol{w}}$ being linearly independents, the above linear system is verified iff $\varphi_{n}=0$ for $n=1 . .31$. 


\section{B Base change relations}

Here we provide the expressions of coefficients $a_{n}$ for $n=1 . .31$, as defined in (34), as functions of the components $A_{i j k p q r}$ :

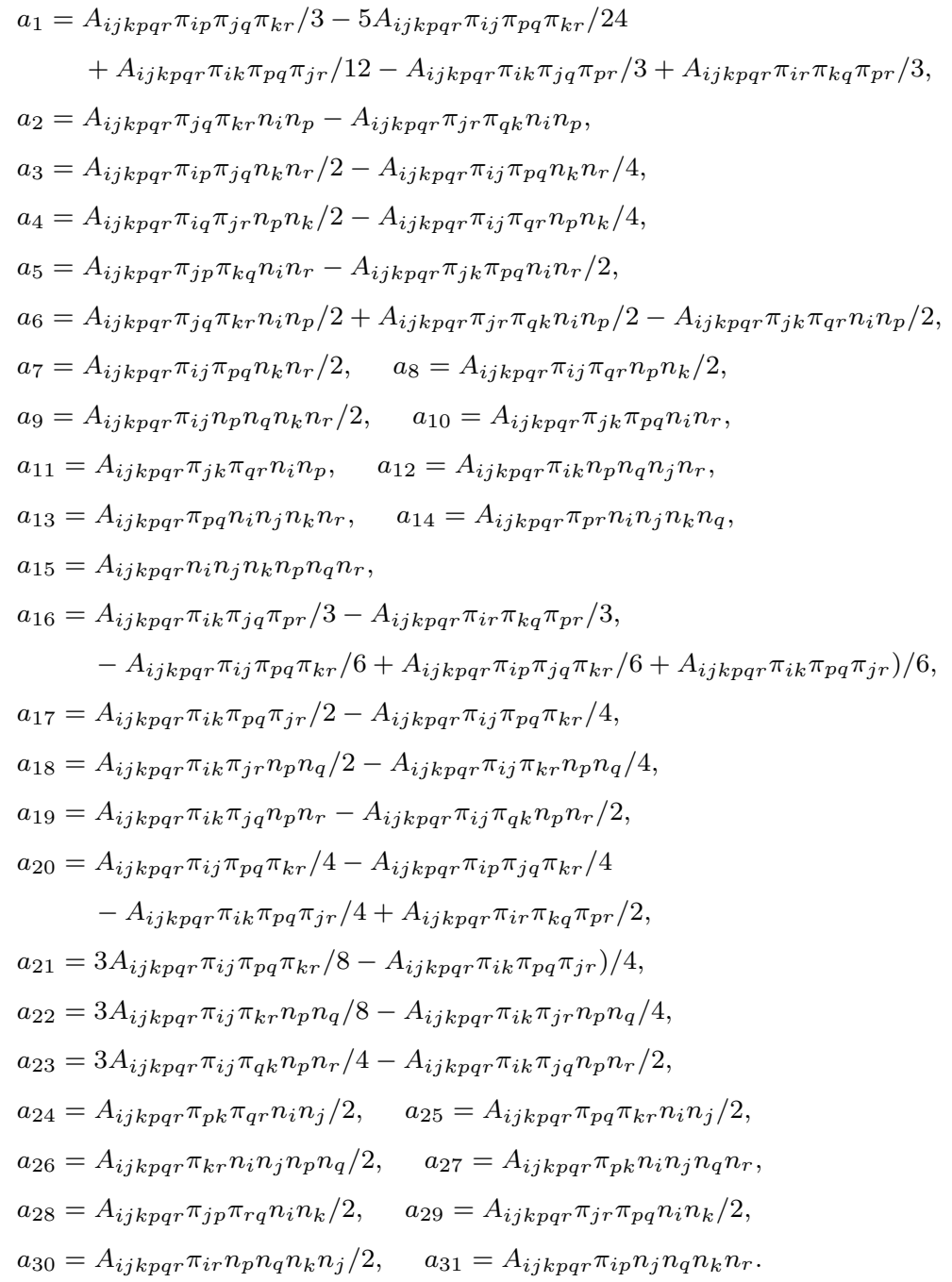




\section{Derivation of the components of the sixth order Eshelby tensor}

The expressions of the harmonic potential $\Phi_{i}$ and of the biharmonic potential $\Psi_{i}$ can be found in Mura [19] (see equations 12.13 and 12.14). They read:

$$
\begin{aligned}
\Phi_{r}= & a_{R}^{2} x_{r} V_{R}, \\
\Psi_{r, i}=- & \frac{1}{4} \delta_{i r} a_{R}^{2}\left\{\left(V-x_{k} x_{k} V_{K}\right)-a_{R}^{2}\left(V_{R}-x_{k} x_{k} V_{R K}\right)\right\} \\
& +a_{R}^{2} x_{r} x_{i}\left(V_{I}-a_{R}^{2} V_{I R}\right) .
\end{aligned}
$$

For an interior point (within the inhomogeneity), $\Phi_{r}$ and $\Psi_{r}$ are polynomial functions respectively of rank 3 and 5 . The sixth order Eshelby tensor is then given by:

$$
\begin{aligned}
8 \pi\left(1-\nu_{0}\right) S_{i j k p q r}^{0} & =\Psi_{r, p q i j k}-2 \nu_{0} \Phi_{r, i j k} \delta_{p q} \\
& -\left(1-\nu_{0}\right)\left(\Phi_{r, j k q} \delta_{i p}+\Phi_{r, i k q} \delta_{j p}+\Phi_{r, j k p} \delta_{i q}+\Phi_{r, i k p} \delta_{j q}\right)
\end{aligned}
$$

where the $V_{i . . j}$ are given, for an interior point, by:

$$
V_{i . . j}=\frac{1}{2}\left(I_{i . . j}-x_{k} x_{k} I_{i . . j K}\right)
$$

and the $I_{i . . j}$ integrals are defined by:

$$
I_{i . . j}=2 \pi a_{1} a_{2} a_{3} \int_{0}^{+\infty} \frac{d s}{\left(a_{i}^{2}+s\right) \ldots\left(a_{j}^{2}+s\right) \Delta(s)} .
$$

The derivatives of $V_{i . . j}$ read (see Mura 1987, eq. 11.40.3):

$$
V_{i . . j, k}=-x_{k} I_{i . . j K}
$$

It can then be shown that:

$$
\begin{gathered}
\Phi_{r, i}=a_{R}^{2} \delta_{i r} V_{R}-a_{R}^{2} x_{r} x_{i} I_{I R}, \\
\Phi_{r, i j}=-a_{R}^{2} \delta_{i r} x_{j} I_{J R}-a_{R}^{2}\left(\delta_{j r} x_{i}+\delta_{i j} x_{r}\right) I_{I R}, \\
\Phi_{r, i j k}=-2 a_{R}^{2} I_{i j k r} I_{I J}-a_{R}^{2} \delta_{i j} \delta_{k r} I_{I R}, \\
\Psi_{r, i j k p q}=\left\{\delta_{i j} \delta_{k r} \delta_{p q} T_{I P R}+2 I_{i j p q} \delta_{k r} T_{I J R}+2 I_{p q k r} \delta_{i j} T_{I K R}\right. \\
+2 I_{i j k r} \delta_{p q} T_{I J P}+2\left(I_{i j p r} \delta_{k q}+I_{i j q r} \delta_{k p}\right) T_{P Q R} \\
\left.+2\left(I_{i j k p} \delta_{q r}+I_{i j k q} \delta_{p r}\right) T_{K P Q}\right\} a_{R}^{2}
\end{gathered}
$$

where the $T_{i j k}$ are related to the $I_{i . . j}$ integrals by

$$
T_{i j k}=I_{i j k} a_{K}^{2}-I_{i j}=I_{i j k} a_{J}^{2}-I_{i k}=I_{i j k} a_{I}^{2}-I_{j k}
$$

The $T_{i j k}$ are invariant by any permutation of the indices $i, j, k$. An integral expression for the $T_{i j k}$ is also given in equation (48). It is obtained by replacing the $I_{i . . j}$ in (67) by their expression given by (64). Finally, from (62) and (66), it is easy to obtain expression (47). 
D Components of the second order Eshelby tensor for spheroidal inhomogeneities

We consider here the case of a spheroidal inhomogeneity. We put $a_{1}=a_{2}=b$ and $a_{3}=a$. By $\epsilon=a / b$ we define the aspect ratio of the spheroid. Expressions for $\mathcal{I}_{i j}$ and $\mathcal{T}_{i j k}$ are

$$
\begin{aligned}
& \mathcal{I}_{11}=\mathcal{I}_{22}=\mathcal{I}_{12}=\frac{\pi\left(3 b^{2}(\eta-1)+2 a^{2}\right)}{2 b^{2}\left(a^{2}-b^{2}\right)}, \mathcal{I}_{13}=\mathcal{I}_{23}=\frac{2 \pi(1-3 \eta)}{a^{2}-b^{2}}, \\
& \mathcal{I}_{33}=\frac{4 \pi\left(3 \eta a^{2}-b^{2}\right)}{3 a^{2}\left(a^{2}-b^{2}\right)} \\
& \mathcal{T}_{111}=\mathcal{T}_{112}=\mathcal{T}_{122}=\mathcal{T}_{222}=\frac{\pi\left(6 a^{2} b^{2}(2-3 \eta)+3 b^{4}(\eta-1)-4 a^{4}\right)}{12 b^{2}\left(a^{2}-b^{2}\right)^{2}}, \\
& \mathcal{T}_{113}=\mathcal{T}_{123}=\mathcal{T}_{223}=\frac{\pi\left(3 b^{2}(\eta-1)+12 \eta a^{2}-2 a^{2}\right)}{2\left(a^{2}-b^{2}\right)^{2}}, \\
& \mathcal{T}_{133}=\mathcal{T}_{233}=\frac{2 \pi\left(b^{2}(5-9 \eta)-6 a^{2} \eta\right)}{3\left(b^{2}-a^{2}\right)^{2}}, \\
& \mathcal{T}_{333}=\frac{4 \pi\left(3 a^{2}(5 \eta-1)-2 b^{2}\right) b^{2}}{15 a^{2}\left(b^{2}-a^{2}\right)^{2}}
\end{aligned}
$$

where $\eta$ is defined by:

$$
\eta= \begin{cases}\frac{a b^{2}}{c^{3}} \operatorname{arctanh}\left\{\frac{c}{a}\right\}-\frac{b^{2}}{c^{2}} & \text { (prolate spheroid) } \\ -\frac{a b^{2}}{c^{3}} \arctan \left\{\frac{c}{a}\right\}+\frac{b^{2}}{c^{2}} & \text { (oblate spheroid) }\end{cases}
$$

The introduction of $\eta$ allows to produce various equations which are valid for both the case of an oblate spheroid $(a \leq b)$ and a prolate spheroid $(b \geq a)$. Due to the symmetries of the problem, the second order Eshelby tensor is transversely isotropic and a representation of this tensor along the basis $\left(\mathbb{T}_{1}, \ldots, \mathbb{T}_{31}\right)$ is then possible:

$$
\mathbb{S}=\sum_{n=1}^{n=31} s_{n} \mathbb{T}_{n}
$$


where the $s_{n}$ for $n=1 . .31$ are defined by:

$$
\begin{aligned}
& s_{1}=\frac{3 \mathcal{T}_{111}+4\left(1-\nu_{0}\right) \mathcal{I}_{11}}{4 \pi\left(1-\nu_{0}\right)} b^{2}, \quad s_{2}=\frac{\mathcal{I}_{13}}{4 \pi} b^{2}, \\
& s_{3}=\frac{\mathcal{T}_{113}+2\left(1-\nu_{0}\right) \mathcal{I}_{13}}{4 \pi\left(1-\nu_{0}\right)} a^{2}, \quad s_{4}=\frac{\mathcal{T}_{113}+\left(1-\nu_{0}\right) \mathcal{I}_{13}}{4 \pi\left(1-\nu_{0}\right)} b^{2}, \\
& s_{5}=\frac{\mathcal{T}_{113}+\left(1-\nu_{0}\right) \mathcal{I}_{13}}{2 \pi\left(1-\nu_{0}\right)} a^{2}, \quad s_{6}=\frac{2 \mathcal{T}_{113}+\left(1-\nu_{0}\right)\left(2 \mathcal{I}_{11}+\mathcal{I}_{13}\right)}{4 \pi\left(1-\nu_{0}\right)} b^{2}, \\
& s_{7}=\frac{\mathcal{T}_{113}+\mathcal{I}_{13}}{2 \pi\left(1-\nu_{0}\right)} a^{2}, \quad s_{8}=\frac{2 \mathcal{T}_{113}+\left(1-\nu_{0}\right) \mathcal{I}_{13}}{4 \pi\left(1-\nu_{0}\right)} b^{2}, \\
& s_{9}=\frac{3 \mathcal{T}_{133}+2 \nu_{0} \mathcal{I}_{13}}{8 \pi\left(1-\nu_{0}\right)} a^{2}, \quad s_{10}=\frac{2 \mathcal{T}_{113}+\left(1+\nu_{0}\right) \mathcal{I}_{13}}{2 \pi\left(1-\nu_{0}\right)} a^{2}, \\
& s_{11}=\frac{4 \mathcal{T}_{113}+\left(1-\nu_{0}\right)\left(4 \mathcal{I}_{11}+\mathcal{I}_{13}\right)}{4 \pi\left(1-\nu_{0}\right)} b^{2}, s_{12}=\frac{3 \mathcal{T}_{133}+2 \mathcal{I}_{13}}{4 \pi\left(1-\nu_{0}\right)} a^{2}, \\
& s_{13}=\frac{3\left[\mathcal{T}_{133}+2 \nu_{0} \mathcal{I}_{33}\right]}{4 \pi\left(1-\nu_{0}\right)} a^{2}, \quad s_{14}=\frac{3 \mathcal{T}_{133}+2 \mathcal{I}_{13}-2 \nu_{0} \mathcal{I}_{13}}{4 \pi\left(1-\nu_{0}\right)} b^{2}, \\
& s_{15}=\frac{3\left[5 \mathcal{T}_{333}+2\left(2-\nu_{0}\right) \mathcal{I}_{33}\right]}{8 \pi\left(1-\nu_{0}\right)} a^{2}, \quad s_{16}=\frac{3 \mathcal{T}_{111}+\left(3-2 \nu_{0}\right) \mathcal{I}_{11}}{2 \pi\left(1-\nu_{0}\right)} b^{2}, \\
& s_{17}=\frac{3 \mathcal{T}_{111}+2 \mathcal{I}_{11}}{2 \pi\left(1-\nu_{0}\right)} b^{2}, \quad s_{18}=\frac{\mathcal{T}_{113}+2 \nu_{0} \mathcal{I}_{11}}{4 \pi\left(1-\nu_{0}\right)} b^{2}, \\
& s_{19}=\frac{\mathcal{T}_{113}+\left(1-\nu_{0}\right) \mathcal{I}_{13}}{2 \pi\left(1-\nu_{0}\right)} a^{2}, \quad s_{20}=\frac{3 \mathcal{T}_{111}+\mathcal{I}_{11}}{4 \pi\left(1-\nu_{0}\right)} b^{2}, \\
& s_{21}=\frac{3 \mathcal{T}_{111}+2 \mathcal{I}_{11}}{4 \pi\left(1-\nu_{0}\right)} b^{2}, \quad s_{22}=\frac{\mathcal{T}_{113}+2 \nu_{0} \mathcal{I}_{11}}{8 \pi\left(1-\nu_{0}\right)} b^{2}, \\
& s_{23}=\frac{\mathcal{T}_{113}}{4 \pi\left(1-\nu_{0}\right)} a^{2}, \quad s_{24}=\frac{2 \mathcal{T}_{113}+\nu_{0} \mathcal{I}_{13}}{4 \pi\left(1-\nu_{0}\right)} b^{2}, \\
& s_{25}=\frac{\mathcal{T}_{113}+\nu_{0} \mathcal{I}_{13}}{2 \pi\left(1-\nu_{0}\right)} b^{2}, \quad s_{26}=\frac{3 \mathcal{T}_{133}+2\left(2-\nu_{0}\right) \mathcal{I}_{13}}{8 \pi\left(1-\nu_{0}\right)} b^{2}, \\
& s_{27}=\frac{3 \mathcal{T}_{133}+2\left(1-\nu_{0}\right) \mathcal{I}_{13}}{4 \pi\left(1-\nu_{0}\right)} a^{2}, \quad s_{28}=\frac{4 \mathcal{T}_{113}+\left(3-\nu_{0}\right) \mathcal{I}_{13}}{8 \pi\left(1-\nu_{0}\right)} b^{2}, \\
& s_{29}=\frac{2 \mathcal{T}_{113}+\left(1+\nu_{0}\right) \mathcal{I}_{13}}{4 \pi\left(1-\nu_{0}\right)} b^{2}, \quad s_{30}=\frac{3 \mathcal{T}_{133}+2 \mathcal{I}_{13}}{8 \pi\left(1-\nu_{0}\right)} b^{2}, \\
& s_{31}=\frac{3 \mathcal{T}_{133}+\left(1-\nu_{0}\right)\left(3 \mathcal{I}_{33}+\mathcal{I}_{13}\right)}{4 \pi\left(1-\nu_{0}\right)} a^{2} .
\end{aligned}
$$

